TRANSACTIONS OF THE

AMERICAN MATHEMATICAL SOCIETY

Volume 350, Number 1, January 1998, Pages 251-274

$\mathrm{S} 0002-9947(98) 02055-8$

\title{
GEOMETRY OF FAMILIES OF NODAL CURVES ON THE BLOWN-UP PROJECTIVE PLANE
}

\author{
GERT-MARTIN GREUEL, CHRISTOPH LOSSEN, AND EUGENII SHUSTIN
}

\begin{abstract}
Let $\mathbb{P}_{r}^{2}$ be the projective plane blown up at $r$ generic points. Denote by $E_{0}, E_{1}, \ldots, E_{r}$ the strict transform of a generic straight line on $\mathbb{P}^{2}$ and the exceptional divisors of the blown-up points on $\mathbb{P}_{r}^{2}$ respectively. We consider the variety $V_{i r r}\left(d ; d_{1}, \ldots, d_{r} ; k\right)$ of all irreducible curves $C$ in $\mid d E_{0}-$ $\sum_{i=1}^{r} d_{i} E_{i} \mid$ with $k$ nodes as the only singularities and give asymptotically nearly optimal sufficient conditions for its smoothness, irreducibility and nonemptiness. Moreover, we extend our conditions for the smoothness and the irreducibility to families of reducible curves. For $r \leq 9$ we give the complete answer concerning the existence of nodal curves in $V_{i r r}\left(d ; d_{1}, \ldots, d_{r} ; k\right)$.
\end{abstract}

\section{INTRODUCTION}

We deal with the following general problem: given a smooth rational surface $S$ and a divisor $D$ on $S$, when is the variety $V_{i r r}(D, k)$ of nodal irreducible curves in the complete linear system $|D|$ with a fixed number $k$ of nodes non-empty, when nonsingular and when irreducible? For $S=\mathbb{P}^{2}$, these questions are completely answered by the classical result of F. Severi ([Sev]), stating that the variety $V_{i r r}(d H, k)$ of irreducible curves of degree $d$ having $k$ nodes is non-empty and smooth exactly if

$$
0 \leq k \leq \frac{(d-1)(d-2)}{2},
$$

and the result of J. Harris ([Har]), stating that $V_{i r r}(d H, k)$ is always irreducible. A modification of Severi's method did lead to a sufficient (smoothness-)criterion for (not necessarily irreducible) curves on general smooth rational surfaces $S$ ([Ta1, Nob]): let $C_{0} \subset S$ be an integral curve, and let $C \in\left|C_{0}\right|$ be a reduced (nodal) curve with precisely $k$ nodes, such that $C=C_{1} \cup \ldots \cup C_{s}$, the $C_{i}$ are irreducible and

$$
K_{S} \cdot C_{i}<0
$$

for each $1 \leq i \leq s$. Then the variety $V\left(\left|C_{0}\right|, C\right)$ of reduced curves $\widetilde{C}=\widetilde{C}_{1} \cup$ $\ldots \cup \widetilde{C}_{s}$, in the linear system $\left|C_{0}\right|$ with precisely $k$ nodes as their only singularities, whose components $\widetilde{C}_{i}$ have the same type (that is, are in the same linear system and have the same number of nodes) as the components of $C$, is smooth (see [Ta2, GrM, GrK, GrL] for generalizations to other surfaces). Moreover, in those cases each node of $C$ can be smoothed independently.

Received by the editors February 1, 1996.

1991 Mathematics Subject Classification. Primary 14H10.

Key words and phrases. Nodal curves, nodes, families of curves, blown-up projective space, existence, equisingular deformation. 
In this paper, we concentrate on the case $S=\mathbb{P}_{r}^{2}$, the projective plane blown up at $r$ generic points $p_{1}, \ldots, p_{r}$. Let $E_{0}, E_{1}, \ldots, E_{r}$ denote the strict transform of a generic straight line on $\mathbb{P}^{2}$ and the exceptional divisors of the blown-up points on $\mathbb{P}_{r}^{2}$, respectively. Then for an irreducible nodal curve $C \in\left|d E_{0}-\sum_{i=1}^{r} d_{i} E_{i}\right|$ the condition (0.0.1) reads as

$$
3 d>\sum_{i=1}^{r} d_{i}
$$

In the blown-down situation, such a curve $C$ corresponds to a plane curve of degree $d$ having (not necessarily ordinary) $d_{i}$-fold points at $p_{i}, 1 \leq i \leq r$, and $k^{\prime} \leq k$ nodes outside. For the variety of irreducible plane curves of fixed topological (or analytic) type, E. Shustin gives in ([Sh2]) an asymptotically improved sufficient condition for the smoothness and the irreducibility:

$$
\alpha d^{2}+o\left(d^{2}\right)>\sum \sigma\left(S_{i}\right)
$$

where $\sigma$ denotes some positive invariant of the singular points. In our case, keeping $k, d$ and the $d_{i}(1 \leq i \leq r)$ fixed, certainly $k^{\prime}$ and the topological types of the multiple points may vary. Nevertheless, we shall obtain sufficient conditions for the smoothness and the irreducibility of the same type, that is, with the same exponent in $d$. Moreover, we can extend them to families of reducible curves and obtain an improvement of the smoothness condition (0.0.1) if $r \gg 0$ and $d \gg 0$. In section 3 , we shall give a complete answer for the existence problem in the case of $r \leq 9$ blownup points (Theorems 3 and 4). For $r \geq 10$, we obtain an exponentially optimal sufficient condition (Corollary 3.1.7), that is, of the same exponent in $d$ as the known restrictions for the existence of the corresponding plane curves with $d_{i}$-fold singularities $S_{i}(1 \leq i \leq r)$ and $k^{\prime}$ nodes $S_{r+1}, \ldots, S_{r+k^{\prime}}$ (from Plücker formulae to inequalities by Varchenko [Var] and Ivinskis [Ivi, HiF]). These restrictions are of type

$$
\alpha_{2} d^{2}+\alpha_{1} d+\alpha_{0}>\sum_{i=1}^{r+k^{\prime}} \sigma\left(S_{i}\right) \quad\left(\alpha_{2}=\text { const }>0\right)
$$

with $\sigma$ some positive invariant depending, at most, quadratically on $d$. Our result improves for $r \gg 0$ and $d \gg 0$ the existence criterion of E. Arbarello and M. Cornalba (in $[\mathrm{ArC}]$ ), which presumed (0.0.1) to be given, and, for the given situation, the only known (general) existence criterion for plane curves with given singularities $S_{1}, \ldots, S_{r+k^{\prime}}($ in $[$ Sh1]):

$$
\frac{(d+3)^{2}}{2} \geq \sum_{i=1}^{r+k^{\prime}}\left(\mu\left(S_{i}\right)+4\right)\left(\mu\left(S_{i}\right)+5\right),
$$

which is not exponentially optimal since the right-hand side may be of order four in $d$. For the proof, we combine a modification of the method of A. Hirschowitz in [Hir] and the smoothing of nodes (cf. [Ta1]).

The main point of interest of our paper is in obtaining sufficient conditions for the smoothness, the irreducibility and the existence, even when (0.0.1) does not apply. For large $r$, our results are (in $d$ ) asymptotically nearly optimal.

We should like to thank the referee for useful comments. 


\section{NOTATION AND TERMINOLOGY}

Throughout this article we consider all objects to be defined over an algebraically closed field $K$ of characteristic zero. We use the following notations:

- $\mathbb{P}_{r}^{2}$ - the projective plane blown up at $r$ generic points $p_{1}, \ldots, p_{r}$.

- $\mathfrak{m}_{z_{\nu}}$ - the maximal ideal in the local ring $\mathcal{O}_{\mathbb{P}_{r}^{2}, z_{\nu}}, z_{\nu} \in \mathbb{P}_{r}^{2}$.

- $E_{0}$ - the strict transform of a generic straight line (in $\mathbb{P}^{2}$ ).

- $E_{i}(1 \leq i \leq r)$ - the exceptional divisor of the blown-up point $p_{i}$ on $\mathbb{P}_{r}^{2}$.

- $V_{i r r}\left(d ; d_{1}, \ldots, d_{r} ; k\right)$ - the variety of all irreducible curves $C$ in the linear system $\left|d E_{0}-\sum_{i=1}^{r} d_{i} E_{i}\right|$ having $\mathrm{k}$ nodes as their only singularities.

Furthermore, for a reduced nodal curve $C \subset \mathbb{P}_{r}^{2}, C=C_{1} \cup \ldots \cup C_{s}\left(C_{i}\right.$ irreducible $)$, having precisely $k$ nodes as their only singularities and a divisor $D$ on $\mathbb{P}_{r}^{2}$, we denote:

- $V(|D| ; C)$ - the variety of all reduced curves $\tilde{C}=\tilde{C}_{1} \cup \ldots \cup \tilde{C}_{s}$ in the linear system $|D|$ with precisely $k$ nodes as their only singularities, whose components $\tilde{C}_{i}$ have the same type (that is, are in the same linear system and have the same number of nodes) as the components $C_{i}(1 \leq i \leq s)$ of $C$.

\section{Smoothness}

1.1. Formulation of the result. For $\mathbb{P}_{r}^{2}, r \leq 8$, condition (0.0.1) is fulfilled for each irreducible curve $C$, hence $V_{i r r}\left(d ; d_{1}, \ldots, d_{r} ; k\right)$ is always smooth. In case $r=9$ and $C \in V_{i r r}\left(d ; d_{1}, \ldots, d_{9} ; k\right),(0.0 .1)$ reads

$$
3 d>\sum_{i=1}^{9} d_{i},
$$

which is satisfied exactly if $C$ is not the (unique) smooth cubic through $p_{1}, \ldots, p_{9}$. Thus $V_{i r r}\left(d ; d_{1}, \ldots, d_{9} ; k\right)$ is always smooth, too. In this section we shall prove:

Theorem 1 (Smoothness Theorem). Let $r \geq 10$, and let the positive integers $d$; $d_{1}, \ldots, d_{r}$ satisfy the two (smoothness) conditions

$$
\begin{aligned}
& {[\sqrt{2 k}]<\frac{d}{2}+3-\frac{\sqrt{2}}{2} \sqrt{\sum_{i=1}^{r}\left(d_{i}+2\right)^{2}},} \\
& {[\sqrt{2 k}]<d+3-\sqrt{2} \sqrt{2+\sum_{i=1}^{r}\left(d_{i}+2\right)\left(d_{i}+1\right)} .}
\end{aligned}
$$

Then $V_{i r r}\left(d ; d_{1}, \ldots, d_{r} ; k\right)$ is smooth and has the $T$-property (that is, each germ of $V_{i r r}\left(d ; d_{1}, \ldots, d_{r} ; k\right)$ is a transversal intersection of germs of equisingular strata corresponding to the $k$ nodes).

Let $C \subset \mathbb{P}_{r}^{2}$ be a reduced nodal curve, $C \in|D|$. If, for each irreducible component $C_{\nu} \in\left|d^{(\nu)} E_{0}-\sum_{i=1}^{r} d_{i}^{(\nu)} E_{i}\right|$ of $C$ (having precisely $k^{(\nu)}$ nodes), the two smoothness conditions are fulfilled, then $V(|D| ; C)$ is smooth and has the T-property.

1.2. Vanishing criteria. We introduce the vanishing criteria which we shall mainly use in the proof of the Smoothness and the Irreducibility Theorem (in the next section): 
For a curve $C \in V_{i r r}\left(d ; d_{1}, \ldots, d_{r} ; k\right)$ and a subset $\Sigma_{0} \subset \operatorname{Sing} C$ we define the sheaf $\mathcal{T}_{C, \Sigma_{0}}^{1}$ to be the skyscraper sheaf concentrated at $\operatorname{Sing} C=\left\{z_{1}, \ldots, z_{k}\right\}$ with stalks

$$
\left(\mathcal{T}_{C, \Sigma_{0}}^{1}\right)_{z_{\nu}}:=\left\{\begin{array}{lll}
\mathcal{O}_{\mathbb{P}_{r}^{2}, z_{\nu}} / \mathfrak{m}_{z_{\nu}} & \text { for } & z_{\nu} \in \operatorname{Sing} C-\Sigma_{0}, \\
\mathcal{O}_{\mathbb{P}_{r}^{2}, z_{\nu}} / \mathfrak{m}_{z_{\nu}}^{2} & \text { for } & z_{\nu} \in \Sigma_{0} .
\end{array}\right.
$$

Furthermore, put $\mathcal{N}_{C / \mathbb{P}_{r}^{2}}^{\Sigma_{0}}:=\operatorname{Ker}\left(\mathcal{N}_{C / \mathbb{P}_{r}^{2}} \longrightarrow \mathcal{T}_{C, \Sigma_{0}}^{1}\right)$.

Proposition 1.2.1. Let $C \in\left|d E_{0}-\sum_{i=1}^{r} d_{i} E_{i}\right|$ be a reduced nodal curve having $k$ nodes as its only singularities, $\tilde{C} \sim d E_{0}-\sum_{i=1}^{r} \tilde{d}_{i} E_{i}$, where $\tilde{d}_{i} \geq d_{i}$ for $1 \leq i \leq r$, and $\Sigma_{0} \subset \operatorname{Sing} C=\left\{z_{1}, \ldots, z_{k}\right\}$. Moreover, let $\tilde{H}$ be a reduced curve whose local equations map to $0 \in\left(\mathcal{T}_{C, \Sigma_{0}}^{1}\right)_{z_{\nu}}$ for $1 \leq \nu \leq k$.

Then $H^{1}\left(C, \mathcal{N}_{C / \mathbb{P}_{r}^{2}}^{\Sigma_{0}}\right)$ vanishes, if the following conditions are satisfied:

(A) $H^{1}\left(\mathbb{P}_{r}^{2}, \mathcal{O}_{\mathbb{P}_{r}^{2}}(C)\right)=0$,

(B) $H^{1}\left(\mathbb{P}_{r}^{2}, \mathcal{O}_{\mathbb{P}_{r}^{2}}(\tilde{C}-\tilde{H})\right)=0$,

(C) $H^{1}\left(\tilde{H}, \tilde{\mathcal{N}}_{\tilde{H} / \mathbb{P}_{r}^{2}}^{\Sigma_{0}}\right)=0$,

where $\tilde{\mathcal{N}}_{\tilde{H} / \mathbb{P}_{r}^{2}}^{\Sigma_{0}}:=\operatorname{Ker}\left(\mathcal{O}_{\mathbb{P}_{r}^{2}}(\tilde{C}) \otimes \mathcal{O}_{\tilde{H}} \longrightarrow \mathcal{T}_{C, \Sigma_{0}}^{1}\right)$.

Proof. We have an exact sequence

$$
\ldots \rightarrow H^{1}\left(\mathbb{P}_{r}^{2}, \mathcal{O}_{\mathbb{P}_{r}^{2}}(C)\right) \rightarrow H^{1}\left(C, \mathcal{N}_{C / \mathbb{P}_{r}^{2}}\right) \rightarrow H^{2}\left(\mathbb{P}_{r}^{2}, \mathcal{O}_{\mathbb{P}_{r}^{2}}\right) \rightarrow \ldots
$$

where, by Serre duality, $H^{2}\left(\mathbb{P}_{r}^{2}, \mathcal{O}_{\mathbb{P}_{r}^{2}}\right)=H^{0}\left(\mathbb{P}_{r}^{2}, K_{\mathbb{P}_{r}^{2}}\right)=0$. Hence, the statement of the proposition follows immediately from the following commutative diagram with exact columns and diagonal

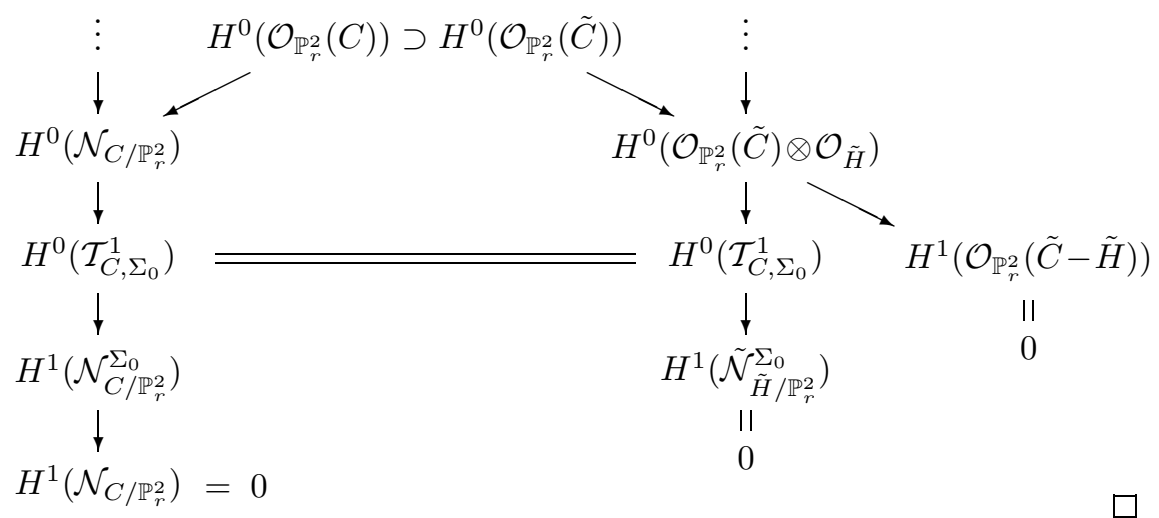

In the following we shall obtain the vanishing properties $(\mathrm{A})-(\mathrm{C})$ by applying two well-known criteria:

Proposition 1.2.2 (Hirschowitz-Criterion, [Hir]). Let $C \sim d E_{0}-\sum_{i=1}^{r} d_{i} E_{i}$, where $d ; d_{1}, \ldots, d_{r}$ are non-negative integers satisfying

$$
\sum_{i=1}^{r} \frac{d_{i}\left(d_{i}+1\right)}{2}<\left[\frac{(d+3)^{2}}{4}\right]
$$

Then $H^{1}\left(\mathbb{P}_{r}^{2}, \mathcal{O}_{\mathbb{P}_{r}^{2}}(C)\right)=0$. 
In a more special situation, let $S_{r}^{I}$ be the projective plane blown up at $r$ points $p_{1}, \ldots, p_{r}$ where $p_{i}, i \in I$, lie on a line, and all the other points are in generic position. Let $C$ be as above such that

$$
\sum_{i \in I} d_{i} \leq d+1
$$

and condition (1.2.3) holds, then $H^{1}\left(S_{r}^{I}, \mathcal{O}_{S_{r}^{I}}(C)\right)=0$.

Proposition 1.2.5 ([GrK]). Let $S$ be a smooth surface, $C \subset S$ a compact reduced curve, $\mathcal{F}$ a torsionfree coherent $\mathcal{O}_{C}$-module which has rank 1 on each irreducible component $C_{i}$ of $C(1 \leq i \leq s)$. Then $H^{1}(C, \mathcal{F})=0$ if for $1 \leq i \leq s$

$$
\chi\left(\overline{\mathcal{F} \otimes \mathcal{O}_{C_{i}}}\right)>\chi\left(\omega_{C} \otimes \mathcal{O}_{C_{i}}\right)-\operatorname{isod}_{C_{i}}\left(\mathcal{F}, \mathcal{O}_{C}\right) .
$$

Here - denotes reduction modulo torsion, $\omega_{C}$ the dualizing sheaf and the isomorphism defect $\operatorname{isod}_{C_{i}}\left(\mathcal{F}, \mathcal{O}_{C}\right)$ is defined to be the sum of all

$$
\operatorname{isod}_{C_{i}, x}\left(\mathcal{F}, \mathcal{O}_{C}\right):=\min \left(\operatorname{dim}_{\mathbb{C}} \operatorname{coker}\left(\varphi_{C_{i}}:\left(\overline{\mathcal{F} \otimes \mathcal{O}_{C_{i}}}\right)_{x} \rightarrow \mathcal{O}_{C_{i}, x}\right)\right),
$$

$x \in C_{i}$, where the minimum is taken over all $\varphi_{C_{i}}$ which are induced by local homomorphisms $\varphi: \mathcal{F}_{x} \rightarrow \mathcal{O}_{C, x}$.

Corollary 1.2.7. If, in the situation of Proposition 1.2.1, we have for each irreducible component $\tilde{H}_{i}(1 \leq i \leq s)$ of $\tilde{H}$

$$
\tilde{H}_{i} \cdot\left(\tilde{C}-\tilde{H}-K_{\mathbb{P}_{r}^{2}}\right)>\#\left(\operatorname{Sing} C \cap \tilde{H}_{i}\right):=\sum_{z \in \operatorname{Sing} C} \text { multiplicity }\left(\tilde{H}_{i}, z\right),
$$

then $H^{1}\left(\tilde{H}, \tilde{\mathcal{N}}_{\tilde{H} / \mathbb{P}_{r}^{2}}^{\Sigma_{0}}\right)$ vanishes.

Proof. Applying the Riemann-Roch Theorem and the adjunction formula, condition (1.2.6) reads

$$
\operatorname{deg}\left(\overline{\tilde{\mathcal{N}}_{\tilde{H} / \mathbb{P}_{r}^{2}}^{\Sigma_{0}} \otimes \mathcal{O}_{\tilde{H}_{i}}}\right)>\left(K_{\mathbb{P}_{r}^{2}}+\tilde{H}\right) \cdot \tilde{H}_{i}-\operatorname{isod}_{\tilde{H}_{i}}\left(\tilde{\mathcal{N}}_{\tilde{H} / \mathbb{P}_{r}^{2}}^{\Sigma_{0}}, \mathcal{O}_{\tilde{H}}\right) .
$$

The exact sequence $0 \rightarrow \tilde{\mathcal{N}}_{\tilde{H} / \mathbb{P}_{r}^{2}}^{\Sigma_{0}} \rightarrow \mathcal{O}_{\mathbb{P}_{r}^{2}}(\tilde{C}) \otimes \mathcal{O}_{\tilde{H}} \rightarrow \mathcal{T}_{C, \Sigma_{0}}^{1} \rightarrow 0$ implies

$$
\operatorname{deg}\left(\overline{\tilde{\mathcal{N}}_{\tilde{H} / \mathbb{P}_{r}^{2}}^{\sum_{0}} \otimes \mathcal{O}_{\tilde{H}_{i}}}\right)=\operatorname{deg}\left(\mathcal{O}_{\mathbb{P}_{r}^{2}}(\tilde{C}) \otimes \mathcal{O}_{\tilde{H}_{i}}\right)-\chi\left(\mathcal{T}_{C, \Sigma_{0}}^{1} \otimes \mathcal{O}_{\tilde{H}_{i}}\right) .
$$

Finally, an easy consideration shows that

$$
\begin{aligned}
\chi\left(\mathcal{T}_{C, \Sigma_{0}}^{1} \otimes\right. & \left.\mathcal{O}_{\tilde{H}_{i}}\right)-\operatorname{isod}_{\tilde{H}_{i}}\left(\tilde{\mathcal{N}}_{\tilde{H} / \mathbb{P}_{r}^{2}}^{\Sigma_{0}}, \mathcal{O}_{\tilde{H}}\right) \\
& =\sum_{z \in \operatorname{Sing} C \cap \tilde{H}_{i}} \operatorname{dim}_{\mathbb{C}}\left(\mathcal{T}_{C, \Sigma_{0}}^{1} \otimes \mathcal{O}_{\tilde{H}_{i}}\right)_{z}-\operatorname{isod}_{\tilde{H}_{i}, z}\left(\tilde{\mathcal{N}}_{\tilde{H} / \mathbb{P}_{r}^{2}}^{\Sigma_{0}}, \mathcal{O}_{\tilde{H}}\right) \\
& \leq \#\left(\operatorname{Sing} C \cap \tilde{H}_{i}\right) .
\end{aligned}
$$

1.3. Proof of the Smoothness Theorem. Following ([GrK], Theorem 6.1), it is sufficient to show that the first cohomology group $H^{1}\left(C, \mathcal{N}_{C / \mathbb{P}_{r}^{2}}^{\emptyset}\right)$ of the sheaf

$$
\mathcal{N}_{C / \mathbb{P}_{r}^{2}}^{\emptyset}=\operatorname{Ker}\left(\mathcal{N}_{C / \mathbb{P}_{r}^{2}} \longrightarrow \mathcal{T}_{C}^{1}\right)
$$

vanishes, where $\mathcal{T}_{C}^{1}$ denotes the skyscraper sheaf concentrated in the singular set Sing $C=\left\{z_{1}, \ldots, z_{k}\right\}$ with stalk in $z_{\nu}$,

$$
\left(\mathcal{T}_{C}^{1}\right)_{z_{\nu}}=\mathcal{O}_{\mathbb{P}_{r}^{2}, z_{\nu}} / \mathfrak{m}_{z_{\nu}}
$$


If the reduced curve $C \subset \mathbb{P}_{r}^{2}$ decomposes as $C=C^{\prime} \cup C^{\prime \prime}$, then we can consider the exact sequence

$$
0 \longrightarrow \mathcal{N}_{C^{\prime} / \mathbb{P}_{r}^{2}} \oplus \mathcal{N}_{C^{\prime \prime} / \mathbb{P}_{r}^{2}} \stackrel{\alpha}{\longrightarrow} \mathcal{N}_{C / \mathbb{P}_{r}^{2}} \longrightarrow \mathcal{O}_{C^{\prime} \cap C^{\prime \prime}} \longrightarrow 0,
$$

$\alpha$ being induced by $\operatorname{id}_{1} \otimes G+F \otimes \mathrm{id}_{2}$, where $F$ (resp. $G$ ) denotes a (local) equation of $C^{\prime}$ (resp. $C^{\prime \prime}$ ). Since $C^{\prime}$ and $C^{\prime \prime}$ intersect only in nodes, $\alpha$ maps precisely $\mathcal{N}_{C^{\prime} / \mathbb{P}_{r}^{2}}^{\emptyset} \oplus \mathcal{N}_{C^{\prime \prime} / \mathbb{P}_{r}^{2}}^{\emptyset}$ to $\mathcal{N}_{C / \mathbb{P}_{r}^{2}}^{\emptyset}$, and the statement of the theorem follows immediately (by induction) from the vanishing statement in the irreducible case.

First, we need some easy considerations about exceptional curves:

Lemma 1.3.1. Let $H \in\left|h E_{0}-\sum_{i=1}^{r} h_{i} E_{i}\right|$ be an irreducible curve, then

$$
\frac{1}{h^{2}} \sum_{i=1}^{r} h_{i}^{2} \leq 2 \text {. }
$$

Proof. The $r$ blown-up points $p_{1}, \ldots, p_{r}$ are chosen generically. Hence, the existence of an irreducible curve $H \in\left|h E_{0}-\sum_{i=1}^{r} h_{i} E_{i}\right|$, that is of an irreducible curve $\bar{H} \subset \mathbb{P}^{2}$ passing through the $p_{i}$ with multiplicity $h_{i}(1 \leq i \leq r)$, implies for an additional point $p_{\nu}^{\prime} \notin H$, close to $p_{\nu}$ with $h_{\nu} \geq 1$, the existence of a curve $\bar{H}^{\prime} \subset \mathbb{P}^{2}$ passing through $p_{i}$ with multiplicity $h_{i}(i \neq \nu)$ and through the additional point $p_{\nu}^{\prime}$ with multiplicity $h_{\nu}$. By Bézout's theorem, the above statement follows immediately.

Remark 1.3.2. We call an irreducible curve $H \in\left|h E_{0}-\sum_{i=1}^{r} h_{i} E_{i}\right|$ an exceptional curve, if

$$
\sum_{i=1}^{r} h_{i}^{2}>h^{2}
$$

Applying Bézout's theorem, it is clear that for fixed data $h, h_{i}(1 \leq i \leq r)$ there is at most one such exceptional curve $H$; hence for fixed degree $h$ there are only finitely many exceptional curves. For example, for $h=1$ the exceptional curves are just the lines connecting two of the blown-up points.

We divide Sing $C=\Sigma_{1} \cup \Sigma_{2}$ where $\Sigma_{2}$ denotes the set of all nodes lying on the exceptional divisors $E_{i}(1 \leq i \leq r)$. Let $H \in\left|h E_{0}-\sum_{i=1}^{r} h_{i} E_{i}\right|$ be a (reduced) curve of minimal degree passing through $\Sigma_{1}$. Such a curve exists (at least) for each $h$ fulfilling $h(h+3) / 2 \geq k$, hence we can suppose $h \leq[\sqrt{2 k}]$. Moreover define

$$
\tilde{H}:=H \cup E_{1} \cup \ldots \cup E_{r} \in\left|h E_{0}-\sum_{i=1}^{r}\left(h_{i}-1\right) E_{i}\right|
$$

and let $\tilde{C} \sim d E_{0}-\sum_{i=1}^{r} \tilde{d}_{i} E_{i}$ with $\tilde{d}_{i}:=\max \left\{d_{i}, h_{i}+\left[\frac{d_{i}}{2}\right]-1\right\}$. Applying Proposition 1.2.1, we have to check three conditions:

(A) By the Hirschowitz-Criterion (1.2.2) $H^{1}\left(\mathbb{P}_{r}^{2}, \mathcal{O}_{\mathbb{P}_{r}^{2}}(C)\right)$ vanishes, because (1.1.2) implies (1.2.3).

(B) The same criterion gives the vanishing of $H^{1}\left(\mathbb{P}_{r}^{2}, \mathcal{O}_{\mathbb{P}_{r}^{2}}(\tilde{C}-\tilde{H})\right)$, because

$$
\begin{aligned}
\sqrt{\frac{(d-h+3)^{2}}{4}} & \geq \frac{d+3-[\sqrt{2 k}]}{2} \stackrel{(1.1 .2)}{>} \sqrt{1+\sum_{i=1}^{r} \frac{\left(d_{i}+1\right)\left(d_{i}+2\right)}{2}} \\
& \geq \sqrt{1+\sum_{i=1}^{r} \frac{\left(\tilde{d}_{i}-h_{i}+1\right)\left(\tilde{d}_{i}-h_{i}+2\right)}{2}}
\end{aligned}
$$


(C) Applying Corollary 1.2.7, we have to check condition (1.2.8) for each irreducible component $H_{\nu} \in\left|h^{(\nu)}-\sum_{i=1}^{r} h_{i}^{(\nu)} E_{i}\right|, 1 \leq \nu \leq s$, of $H$ and each exceptional divisor $E_{i}, 1 \leq i \leq r$ :

$$
\begin{aligned}
& E_{i} \cdot\left(\tilde{C}-\tilde{H}-K_{\mathbb{P}_{r}^{2}}\right)-\#\left(\operatorname{Sing} C \cap E_{i}\right) \stackrel{\text { Bézout }}{\geq} \tilde{d}_{i}-\left(h_{i}-1\right)+1-\left[\frac{d_{i}}{2}\right] \\
& >0 \\
& H_{\nu} \cdot\left(\tilde{C}-\tilde{H}-K_{\mathbb{P}_{r}^{2}}\right)-\#\left(\operatorname{Sing} C \cap H_{\nu}\right) \\
& \quad \stackrel{\text { Bézout }}{\geq} h^{(\nu)}(d-h+3)-\sum_{i=1}^{r} h_{i}^{(\nu)}\left(\tilde{d}_{i}-\left(h_{i}-1\right)+1\right)-\left[\frac{h^{(\nu)} d-\sum_{i=1}^{r} h_{i}^{(\nu)} d_{i}}{2}\right] \\
& \quad \text { Cauchy } h^{(\nu)}\left(\frac{d}{2}+3-[\sqrt{2 k}]\right)-\sqrt{\sum_{i=1}^{r}\left(h_{i}^{(\nu)}\right)^{2}} \sqrt{\sum_{i=1}^{r} \frac{\left(d_{i}+2\right)^{2}}{4}} \\
& \quad \stackrel{(1.3 .1)}{\geq} h^{(\nu)}\left(\frac{d}{2}+3-[\sqrt{2 k}]-\frac{\sqrt{2}}{2} \sqrt{\sum_{i=1}^{r}\left(d_{i}+2\right)^{2}}\right)^{(1.1 .1)} 0 .
\end{aligned}
$$

\section{IRREDUCIBILITY}

2.1. Formulation of the result. For $\mathbb{P}_{1}^{2}$, the projective plane blown up at one point $p_{1}$, Z. Ran shows in [Ran] that the variety of all irreducible nodal curves $C \in$ $\left|d E_{0}-d_{1} E_{1}\right|$ having exactly $k$ nodes, none of them lying on the exceptional divisor $E_{1}$, is irreducible. Using the smoothness of $V_{i r r}\left(d ; d_{1} ; k\right)$, one can easily deduce its irreducibility. The aim of this section is to prove the following irreducibility criterion for $V_{i r r}\left(d ; d_{1}, \ldots, d_{r} ; k\right)(r \geq 2)$ :

Theorem 2 (Irreducibility Theorem). Let $r \geq 2$, and let the positive integers $d$; $d_{1}, \ldots, d_{r}$ satisfy the two (irreducibility) conditions

$$
\begin{aligned}
& {[\sqrt{2 k}]<\frac{d}{4}+1-\frac{1}{4} \sqrt{\sum_{i=1}^{r} d_{i}^{2}},} \\
& {[\sqrt{2 k}]<\frac{d}{2}+1-\frac{\sqrt{2}}{2} \sqrt{\sum_{i=1}^{r}\left(d_{i}+2\right)^{2}} .}
\end{aligned}
$$

Then $V_{i r r}\left(d ; d_{1}, \ldots, d_{r} ; k\right)$ is (smooth and) irreducible.

Let $C \subset \mathbb{P}_{r}^{2}$ be a reduced nodal curve, $C \in|D|$. If for each irreducible component $C_{\nu} \in\left|d^{(\nu)} E_{0}-\sum_{i=1}^{r} d_{i}^{(\nu)} E_{i}\right|, 1 \leq \nu \leq s$, of $C$ (having precisely $k^{(\nu)}$ nodes) the variety $V_{\text {irr }}\left(d^{(\nu)} ; d_{1}^{(\nu)}, \ldots, d_{r}^{(\nu)} ; k^{(\nu)}\right)$ is smooth and irreducible, then $V(|D| ; C)$ is irreducible.

The main idea of our proof is as follows. We show that for an irreducible curve $C \in\left|d E_{0}-\sum_{i=1}^{r} d_{i} E_{i}\right|$ in an open dense subset $\tilde{V} \subset V_{i r r}\left(d ; d_{1}, \ldots, d_{r} ; k\right)$ the cohomology group $H^{1}\left(C, \mathcal{N}_{C / \mathbb{P}_{r}^{2}}^{\operatorname{Sing} C}\right)$ vanishes (cf. Section 1.2), especially, that the conditions imposed by fixing the $k$ singular points are independent. It follows that the restricted morphism

$$
\begin{aligned}
\pi_{\tilde{V}}: \tilde{V} & \longrightarrow \operatorname{Sym}^{k}\left(\mathbb{P}_{r}^{2}\right) \\
C & \longmapsto \operatorname{Sing} C
\end{aligned}
$$


is dominant; its fibres are all equidimensional and irreducible as open subsets of the linear system $H^{0}\left(\mathbb{P}_{r}^{2}, \operatorname{Ker}\left(\mathcal{O}\left(d E_{0}-\sum d_{i} E_{i}\right) \rightarrow \mathcal{T}_{C, \operatorname{Sing} C}^{1}\right)\right)$. Hence $\tilde{V}$ is irreducible, which implies the irreducibility of $V_{i r r}\left(d ; d_{1}, \ldots, d_{r} ; k\right)$. The second statement is a consequence of the fact that for a fixed reduced nodal curve $C$ as above, each generic member $\tilde{C}$ of a component of $V(|D| ; C)$ decomposes into components $\tilde{C}_{\nu}$, $1 \leq \nu \leq s$, which are generic elements of $V_{i r r}\left(d^{(\nu)} ; d_{1}^{(\nu)}, \ldots, d_{r}^{(\nu)} ; k^{(\nu)}\right)$. Hence there is a well-defined dominant morphism

$$
\prod_{\nu=1}^{n} U_{\nu} \longrightarrow V(|D| ; C)
$$

where $U_{\nu}$ is open dense in $V_{i r r}\left(d^{(\nu)} ; d_{1}^{(\nu)}, \ldots, d_{r}^{(\nu)} ; k^{(\nu)}\right)$.

2.2. Proof of the Irreducibility Theorem. We start the proof by defining the subset $\tilde{V}$ of $V_{i r r}\left(d ; d_{1}, \ldots, d_{r} ; k\right)$ as the set of all irreducible curves $C$ in $V_{i r r}\left(d ; d_{1}, \ldots, d_{r} ; k\right)$ having the subsequent properties:

(a) $\operatorname{Sing} C \cap E_{i}=\emptyset$ for $i=1, \ldots, r$.

(b) If $E$ is an exceptional curve of degree $e \leq 2 k$ then $\operatorname{Sing} C \cap E=\emptyset$.

(c) The $k$ nodes of $C$ are in general position, that is, if $H \in\left|h E_{0}-\sum_{i=1}^{r} h_{i} E_{i}\right|$ is a curve containing Sing $C$ and $H_{\nu} \in\left|h^{(\nu)} E_{0}-\sum_{i=1}^{r} h_{i}^{(\nu)} E_{i}\right|$ is an irreducible component of $H$, then $h^{(\nu)}\left(h^{(\nu)}+3\right) / 2 \geq k_{\nu}:=\#\left(\operatorname{Sing} C \cap H_{\nu}\right)$.

Remark 2.2.1. Condition (c) implies the existence of an irreducible curve among all curves $H \in\left|h E_{0}-\sum_{i=1}^{r} h_{i} E_{i}\right|$ of minimal degree containing Sing $C$ : Assume a curve $H=H_{1} \cup H_{2} \cup \tilde{H}$ of minimal degree $h$ decomposes $\left(h^{(1)} \leq h^{(2)}\right)$, then we know that $H_{1} \cup H_{2}$ contains at most

$$
\begin{aligned}
& \frac{h^{(1)}\left(h^{(1)}+3\right)}{2}+\frac{h^{(2)}\left(h^{(2)}+3\right)}{2} \\
& \quad=\frac{\left(h^{(1)}+h^{(2)}-1\right)\left(h^{(1)}+h^{(2)}+2\right)}{2}-\frac{h^{(1)}\left(h^{(2)}-2\right)+h^{(2)}\left(h^{(1)}-2\right)}{2}+1
\end{aligned}
$$

nodes of $C$. The degree of $H$ being minimal, we conclude that either $h^{(1)}=1$ or $h^{(1)}=h^{(2)}=2$. But in these cases, using the obvious constructions and Bertini's theorem, we can show the existence of an irreducible curve of degree $h^{(1)}+h^{(2)}$ which contains the nodes of $C$ lying on $H_{1} \cup H_{2}$.

Lemma 2.2.2. $\tilde{V} \subset V_{\text {irr }}\left(d ; d_{1}, \ldots, d_{r} ; k\right)$ is an open dense subset.

Proof. The openness being obvious, it is enough to show that there are no obstructions for (locally) moving singular points of $C \in V_{i r r}\left(d ; d_{1}, \ldots, d_{r} ; k\right)$ in a prescribed position (such that the conditions (a)-(c) are satisfied). Again, we divide Sing $C=\Sigma_{1} \cup \Sigma_{2}$ where $\Sigma_{2}$ denotes the set of all nodes lying on the exceptional divisors $E_{i}(1 \leq i \leq r)$ and start moving nodes away from the exceptional divisors (such that finally $\Sigma_{2}=\emptyset$ ):

Let $z$ be a node of $C$ on $E_{i_{0}}$, we have to show that for $\Sigma_{0}:=\operatorname{Sing} C-\{z\}$ the cohomology group $H^{1}\left(C, \mathcal{N}_{C / \mathbb{P}_{r}^{2}}^{\Sigma_{0}}\right)$ vanishes. Indeed, from the commutative diagram 


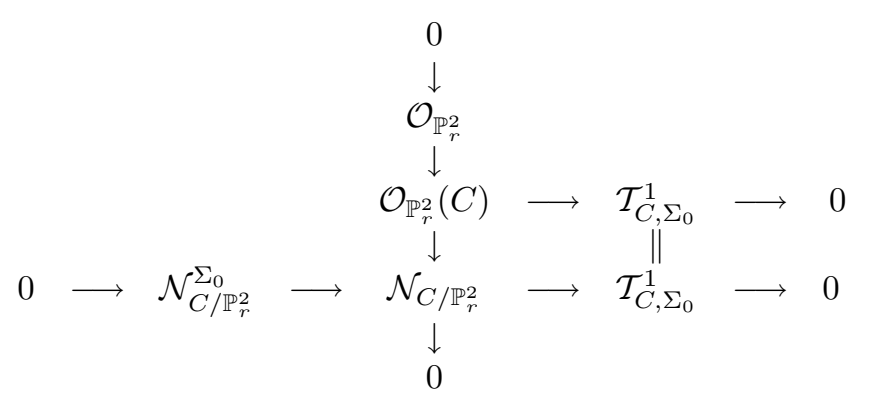

we can conclude the required surjectivity of $H^{0}\left(\mathbb{P}_{r}^{2}, \mathcal{O}_{\mathbb{P}_{r}^{2}}(C)\right) \rightarrow H^{0}\left(\mathbb{P}_{r}^{2}, \mathcal{T}_{C, \Sigma_{0}}^{1}\right)$.

As above, we denote by $H \in\left|h E_{0}-\sum_{i=1}^{r} h_{i} E_{i}\right|$ a curve of minimal degree $h \leq[\sqrt{2 k}]$ passing through $\Sigma_{1}$. There are two cases to consider:

Case 1: $\{z\} \not \subset H$.

Define $L$ to be the strict transform of a straight line $\bar{L} \subset \mathbb{P}^{2}$ through $p_{i_{0}}$ with tangent direction corresponding to $z$ and denote $J:=\left\{j \mid\left\{p_{j}\right\} \subset \bar{L}\right\} \supset\left\{i_{0}\right\}$ (the genericity of the blown-up points implies that $\# J \leq 2$ ). Consider the curve $\tilde{H}:=H \cup E_{1} \cup \ldots \cup E_{r} \cup L \in\left|(h+1) E_{0}-\sum_{i=1}^{r}\left(\tilde{h}_{i}-1\right) E_{i}\right|$, where

$$
\tilde{h}_{i}:=\left\{\begin{array}{ccc}
h_{i}+1 & & i \in J \\
h_{i} & \text { for } & i \notin J .
\end{array}\right.
$$

Moreover, let $\tilde{C} \sim d E_{0}-\sum_{i=1}^{r} \tilde{d}_{i} E_{i}$ with

$$
\tilde{d}_{i}:=\max \left\{d_{i}, \tilde{h}_{i}+\left[\frac{d_{i}}{2}\right]-1\right\}
$$

According to Proposition 1.2.1 we have three conditions, sufficient for the vanishing of $H^{1}\left(C, \mathcal{N}_{C / \mathbb{P}_{r}^{2}}^{\Sigma_{0}}\right)$ : as above, the Hirschowitz-Criterion (1.2.2) together with (2.1.2) guarantees the vanishing property (A). (B) follows in the same manner, because

$$
\begin{aligned}
\sqrt{\frac{(d-h+2)^{2}}{4}} & \geq \frac{d+2-[\sqrt{2 k}]}{2} \stackrel{(2.1 .2)}{>} \sqrt{\sum_{i=1}^{r} \frac{\left(d_{i}+2\right)^{2}}{2}} \\
& \geq \sqrt{1+\sum_{i=1}^{r} \frac{\left(\tilde{d}_{i}-\tilde{h}_{i}+1\right)\left(\tilde{d}_{i}-\tilde{h}_{i}+2\right)}{2}}
\end{aligned}
$$

Finally, property $(\mathrm{C})$ is an immediate consequence of Corollary 1.2.7, knowing that for each exceptional divisor $E_{i}(1 \leq i \leq r)$ we have

$$
E_{i} \cdot\left(\tilde{C}-\tilde{H}-K_{\mathbb{P}_{r}^{2}}\right)-\#\left(\operatorname{Sing} C \cap E_{i}\right) \stackrel{\text { Bézout }}{\geq} \tilde{d}_{i}-\left(\tilde{h}_{i}-1\right)+1-\left[\frac{d_{i}}{2}\right]>0
$$


and that for each irreducible component $H_{\nu} \in\left|h^{(\nu)}-\sum_{i=1}^{r} h_{i}^{(\nu)} E_{i}\right|$ of $L \cup H$

$$
\begin{aligned}
H_{\nu} \cdot\left(\tilde{C}-\tilde{H}-K_{\mathbb{P}_{r}^{2}}\right)-\#\left(\operatorname{Sing} C \cap H_{\nu}\right) \\
\quad \stackrel{\text { Bézout }}{\geq} h^{(\nu)}(d-(h+1)+3)-\sum_{i=1}^{r} h_{i}^{(\nu)}\left(\tilde{d}_{i}-\left(\tilde{h}_{i}-1\right)+1\right)-\left[\frac{h^{(\nu)} d-\sum h_{i}^{(\nu)} d_{i}}{2}\right] \\
\stackrel{\text { Cauchy }}{\geq} h^{(\nu)}\left(\frac{d}{2}+2-[\sqrt{2 k}]\right)-\sqrt{\sum_{i=1}^{r}\left(h_{i}^{(\nu)}\right)^{2}} \sqrt{\sum_{i=1}^{r} \frac{\left(d_{i}+2\right)^{2}}{4}} \\
\quad \stackrel{(1.3 .1)}{\geq} h^{(\nu)}\left(\frac{d}{2}+2-[\sqrt{2 k}]-\frac{\sqrt{2}}{2} \sqrt{\sum_{i=1}^{r}\left(d_{i}+2\right)^{2}}\right) \stackrel{(2.1 .2)}{>} 0 .
\end{aligned}
$$

Case 2: $\{z\} \subset H$.

In this case we can omit the additional component $L$ in the definition of $\tilde{H}$ and, proceeding as in Case 1, we obtain again the vanishing of $H^{1}\left(C, \mathcal{N}_{C / \mathbb{P}_{r}^{2}}^{\Sigma_{0}}\right)$.

Now, we can assume $\operatorname{Sing} C=\Sigma_{1}$ (that is, there are no nodes of $C$ on the exceptional divisors $E_{i}$ ) and we go on moving, subsequently, nodes away from exceptional curves:

Assume $E \in\left|e E_{0}-\sum_{i=1}^{r} e_{i} E_{i}\right|$ to be an irreducible curve satisfying $\sum_{i=1}^{r} e_{i}^{2}>$ $e^{2}$, let $z \in \Sigma_{1}$ be a node of $C$ on $E$ and denote $\Sigma_{0}:=\operatorname{Sing} C-\{z\}$. As above, we construct a curve

$$
\tilde{H}:=H \cup \tilde{L} \in\left|(h+1) E_{0}-\sum_{i=1}^{r} h_{i} E_{i}\right|
$$

where $\tilde{L} \not \subset H$ corresponds to a line in $\mathbb{P}^{2}$ containing none of the blown-up points such that $\tilde{L} \cap \operatorname{Sing} C=\{z\}$. Moreover, we consider $\tilde{C} \sim d E_{0}-\sum_{i=1}^{r} \tilde{d}_{i} E_{i}$ with

$$
\tilde{d}_{i}:=\max \left\{d_{i}, h_{i}+\left[\frac{d_{i}}{2}\right]\right\},
$$

and the above reasoning again gives $H^{1}\left(C, \mathcal{N}_{C / \mathbb{P}_{r}^{2}}^{\Sigma_{0}}\right)=0$.

By Remark 1.3.2, we can end up with a curve $C \in V_{i r r}\left(d ; d_{1}, \ldots, d_{r} ; k\right)$ close to the original one having properties (a) and (b). It remains to move the nodes into general position to obtain a curve $C \in \tilde{V}$ :

Again, we choose a (not necessarily irreducible) curve $H \in\left|h E_{0}-\sum_{i=1}^{r} h_{i} E_{i}\right|$ of minimal degree containing $\Sigma_{1}=\operatorname{Sing} C$. Assume $H$ decomposes into irreducible components $H_{\nu} \in\left|h^{(\nu)}-\sum_{i=1}^{r} h_{i}^{(\nu)} E_{i}\right|(1 \leq \nu \leq s)$ and assume that there are more than $M:=h^{(\nu)}\left(h^{(\nu)}+3\right) / 2+1$ nodes on one component $H_{\nu}$. We show that we can move $M$ of them, $z_{1}, \ldots, z_{M}$, into general position:

Define $\tilde{H}:=H \cup G$ where $G \in\left|g E_{0}-\sum_{i=1}^{r} g_{i} E_{i}\right|$ is a curve not containing $H_{\nu}$ and passing through $z_{1}, \ldots, z_{M}$. Such a curve exists for each $g$ satisfying

$$
\frac{g(g+3)}{2}-M>\frac{\left(g-h^{(\nu)}\right)\left(g-h^{(\nu)}+3\right)}{2}
$$

(the right-hand side is the dimension of the linear system of curves containing $H_{\nu}$ ). Hence, we can suppose $g=h^{(\nu)}+2$, and $M \leq k$ implies $h^{(\nu)} \leq[\sqrt{2 k}]-1$. Proceeding as before, we have to prove the vanishing of $H^{1}\left(\mathbb{P}_{r}^{2}, \mathcal{O}_{\mathbb{P}_{r}^{2}}(C-\tilde{H})\right)$ and of $H^{1}\left(\tilde{H}, \tilde{\mathcal{N}}_{\tilde{H} / \mathbb{P}_{r}^{2}}^{\Sigma_{0}}\right), \Sigma_{0}=\operatorname{Sing} C-\left\{z_{1}, \ldots, z_{M}\right\}$. The first is an immediate consequence 
of the Hirschowitz-Criterion (1.2.2), because

$$
\begin{aligned}
\sqrt{\frac{(d-h-g+3)^{2}}{4}} & \geq \frac{d-2[\sqrt{2 k}]+2}{2} \\
& \stackrel{(2.1 .2)}{>} \sqrt{\sum_{i=1}^{r} \frac{\left(d_{i}+2\right)^{2}}{2}} \geq \sqrt{1+\sum_{i=1}^{r} \frac{\left(d_{i}-h_{i}-g_{i}\right)\left(d_{i}-h_{i}-g_{i}+1\right)}{2}}
\end{aligned}
$$

while the second results from Corollary 1.2.7, knowing that $H_{\nu}(1 \leq \nu \leq s)$ and the components of $G$ are not exceptional curves:

$$
\begin{aligned}
& H_{\nu} \cdot\left(C-\tilde{H}-K_{\mathbb{P}_{r}^{2}}\right)-\#\left(\operatorname{Sing} C \cap H_{\nu}\right) \\
& \quad \stackrel{\text { Bézout }}{\geq} h^{(\nu)}(d-h-g+3)-\sum_{i=1}^{r} h_{i}^{(\nu)}\left(d_{i}-h_{i}-g_{i}+1\right)-\left[\frac{h^{(\nu)} d-\sum_{i=1}^{r} h_{i}^{(\nu)} d_{i}}{2}\right] \\
& \left.\quad \stackrel{\sqrt{\sum_{i=1}^{r} d_{i}^{2}}}{2}\right) \stackrel{(2.1 .1)}{>} 0
\end{aligned}
$$

and the same holds for each irreducible component $G_{\nu}$ of $G$ in place of $H_{\nu}$.

Lemma 2.2.3. Let $C \in \tilde{V}$, then $H^{1}\left(C, \mathcal{N}_{C / \mathbb{P}_{r}^{2}}^{\operatorname{Sing} C}\right)=0$.

Proof. By Remark 2.2.1, we can choose an irreducible curve $H \in\left|h E_{0}-\sum_{i=1}^{r} h_{i} E_{i}\right|$ of degree $h=[\sqrt{2 k}]$ through all nodes of $C$. Moreover, there is a curve $G \not \supset H$, $G \in\left|g E_{0}-\sum_{i=1}^{r} g_{i} E_{i}\right|$, such that $\operatorname{Sing} C \subset G \cap H$, for each $g$ satisfying

$$
\frac{g(g+3)}{2}-k>\frac{(g-h)(g-h+3)}{2},
$$

hence, especially for $g=h+1$. Let $\tilde{H}:=H \cup G$. Applying Proposition 1.2.1 as before, we conclude the vanishing of $H^{1}\left(C, \mathcal{N}_{C / \mathbb{P}_{r}^{2}}^{\operatorname{Sing} C}\right)$. Indeed, the above inequalities hold again, since neither $H$ nor the irreducible components $G_{\nu}$ of $G$ are exceptional curves.

\section{EXISTENCE}

3.1. Formulation of the result. We shall treat the problem of the existence of nodal curves in $\mathbb{P}_{r}^{2}$ for $r \leq 9$ and $r \geq 10$ separately. If $r \leq 9$, Theorems 3 and 4 will give the complete answer, while for $r \geq 10$, we obtain an asymptotically nearly optimal sufficient criterion (Theorem 5).

Theorem 3 (Existence Theorem A). Let $r=1$, then $V_{\text {irr }}\left(d ; d_{1} ; k\right) \neq \emptyset$ if and only if $\left(d_{1} \leq d-1\right.$ or $\left.d=d_{1}=1\right)$ and

$$
0 \leq k \leq \frac{(d-1)(d-2)-d_{1}\left(d_{1}-1\right)}{2} .
$$

Let $r=2$, and let $d, d_{1}, d_{2}$ be positive integers, then $V_{i r r}\left(d ; d_{1}, d_{2} ; k\right) \neq \emptyset$ if and only if

$$
0 \leq k \leq \frac{(d-1)(d-2)-d_{1}\left(d_{1}-1\right)-d_{2}\left(d_{2}-1\right)}{2}
$$

and either $\left(d_{1}+d_{2} \leq d\right)$ or $\left(d=d_{1}=d_{2}=1\right)$. 
Let $3 \leq r \leq 9$, then we define two $(r+1)$-tuples $\left(d ; d_{1}, \ldots, d_{r}\right)$ and $\left(\tilde{d} ; \tilde{d}_{1}, \ldots, \tilde{d}_{r}\right)$ of non-negative integers to be equivalent, if there is a finite sequence of Cremona maps and a permutation $\sigma$ transforming $\left(d ; d_{1}, \ldots, d_{r}\right)$ to $\left(\tilde{d} ; \tilde{d}_{\sigma(1)}, \ldots, \tilde{d}_{\sigma(r)}\right)$. Here, by a Cremona map, we denote a mapping

$$
\begin{array}{rlcc}
\Sigma_{j, \ell, n}: & \mathbb{Z}^{r+1} & \longrightarrow & \mathbb{Z}^{r+1} \\
& \left(d ; d_{1}, \ldots, d_{r}\right) & \mapsto & \left(d^{\prime} ; d_{1}^{\prime}, \ldots, d_{r}^{\prime}\right)
\end{array}
$$

with

$$
\begin{aligned}
& d^{\prime}=2 d-d_{j}-d_{\ell}-d_{n}, \quad d_{i}^{\prime}=d_{i} \text { for each } i \notin\{j, \ell, n\}, \\
& d_{j}^{\prime}=d-d_{\ell}-d_{n}, d_{\ell}^{\prime}=d-d_{j}-d_{n} \text { and } d_{n}^{\prime}=d-d_{j}-d_{\ell} .
\end{aligned}
$$

Such a Cremona map corresponds to the standard Cremona transformation in $\mathbb{P}^{2}$ inducing the base change in $\operatorname{Pic}\left(\mathbb{P}_{r}^{2}\right)$ :

$$
\left\{\begin{array}{l}
E_{0}^{\prime}=2 E_{0}-E_{j}-E_{\ell}-E_{n} \\
E_{j}^{\prime}=E_{0}-E_{\ell}-E_{n} \\
E_{\ell}^{\prime}=E_{0}-E_{j}-E_{n} \\
E_{n}^{\prime}=E_{0}-E_{j}-E_{\ell} \\
E_{i}^{\prime}=E_{i} \text { for each } i \notin\{j, \ell, n\} .
\end{array}\right.
$$

Since the Cremona transformation preserves the generality of the blown-up points, such a transformation maps elements in $V_{i r r}\left(d ; d_{1}, \ldots, d_{r} ; k\right)$ to elements in $V_{i r r}\left(d^{\prime} ; d_{1}^{\prime}, \ldots, d_{r}^{\prime} ; k\right)$ supposing $d, d^{\prime}, d_{i}, d_{i}^{\prime}(1 \leq i \leq r)$ to be non-negative. We deduce that the non-emptiness of $V_{i r r}\left(d ; d_{1}, \ldots, d_{r} ; k\right)$ is equivalent to the existence of a curve in $V_{i r r}\left(d^{\prime} ; d_{1}^{\prime}, \ldots, d_{r}^{\prime} ; k\right)$. An (ordered) tuple $\left(d ; d_{1}, \ldots, d_{r}\right) \in \mathbb{N}^{r+1}$, $d_{1} \geq d_{2} \geq \ldots \geq d_{r}$, is called minimal, if it satisfies the (minimality) condition

$$
\max _{\#\{j, \ell, n\}=3}\left(d_{j}+d_{\ell}+d_{n}\right)=d_{1}+d_{2}+d_{3} \leq d .
$$

Theorem 4 (Existence Theorem B). Let $3 \leq r \leq 9$, and let positive integers $d \geq$ $d_{1} \geq \ldots \geq d_{r}$ satisfy the condition

$$
\sum_{i=1}^{r} d_{i} \leq 3 d-1
$$

Then $V_{i r r}\left(d ; d_{1}, \ldots, d_{r} ; k\right) \neq \emptyset$ if and only if

$$
0 \leq k \leq \frac{(d-1)(d-2)}{2}-\sum_{i=1}^{r} \frac{d_{i}\left(d_{i}-1\right)}{2}
$$

and $\left(d ; d_{1}, \ldots, d_{r}\right)$ is equivalent to a minimal tuple $\left(\tilde{d} ; \tilde{d}_{1}, \ldots, \tilde{d}_{r}\right)$ of non-negative integers or to the tuple $(1 ; 1,1,0, \ldots, 0)$.

Remark 3.1.4. (A) Condition (3.1.3) is necessary in the following sense. By the Bézout theorem and the generality of the blown-up points, the only type of an irreducible curve not satisfying (3.1.3) is the smooth cubic through the 9 generic points. 
(B) For $3 \leq r \leq 8$ the tuple $\left(d ; d_{1}, \ldots, d_{r}\right)$ is equivalent to a minimal one exactly if the following conditions are satisfied

$$
\begin{aligned}
d & \geq d_{1}+d_{2} \\
2 d & \geq d_{1}+d_{2}+d_{3}+d_{4}+d_{5} \\
3 d & \geq 2 d_{1}+d_{2}+d_{3}+d_{4}+d_{5}+d_{6}+d_{7} \\
4 d & \geq 2 d_{1}+2 d_{2}+2 d_{3}+d_{4}+d_{5}+d_{6}+d_{7}+d_{8} \\
5 d & \geq 2 d_{1}+2 d_{2}+2 d_{3}+2 d_{4}+2 d_{5}+2 d_{6}+d_{7}+d_{8} \\
6 d & \geq 3 d_{1}+2 d_{2}+2 d_{3}+2 d_{4}+2 d_{5}+2 d_{6}+2 d_{7}+2 d_{8}
\end{aligned}
$$

(C) The exceptional case $\left(d ; d_{1}, \ldots, d_{r}\right) \sim(1 ; 1,1,0, \ldots, 0)$ corresponds exactly to the exceptional curves with data

$(2 ; 1,1,1,1,1)$

$(3 ; 2,1,1,1,1,1,1)$

$(4 ; 2,2,2,1,1,1,1,1)$

$(5 ; 2,2,2,2,2,2,1,1)$

$(6 ; 3,2,2,2,2,2,2,2) \quad$ the sextic having nodes at 7 of the generic points

the conic through 5 of the generic points,

the cubic through 7 of the generic points having a node at one of them,

the quartic through 8 generic points having nodes at three of them,

the quintic through all 8 generic points having nodes at 6 of them, and a triple point at the remaining one.

For the proof, using Cremona transformations, we already saw that we can reduce the existence problem to the case of minimal data. Due to the independence of node smoothings in the case $r \leq 9$, it will then be enough to construct only rational curves, i.e. with

$$
0 \leq k=\frac{(d-1)(d-2)}{2}-\sum_{i=1}^{r} \frac{d_{i}\left(d_{i}-1\right)}{2}
$$

nodes, in case (3.1.3) and the minimality condition (3.1.2) are satisfied.

Theorem 5 (Existence Theorem C). Let $r \geq 10$, and let the positive integers $d, d^{\prime}$; $d_{1}, \ldots, d_{r}$ satisfy $d \geq d^{\prime}$ and

$$
\frac{d^{\prime 2}+6 d^{\prime}-1}{4}-\left[\frac{d^{\prime}}{2}\right]>\sum_{i=1}^{r} \frac{d_{i}\left(d_{i}+1\right)}{2} .
$$

Then for any integer $k$ such that

$$
0 \leq k \leq \frac{(d-1)(d-2)}{2}-\frac{\left(d^{\prime}-1\right)\left(d^{\prime}-2\right)}{2},
$$

there exists a reduced irreducible curve $C$ in the linear system $\left|d E_{0}-\sum_{i=1}^{r} d_{i} E_{i}\right|$ on $\mathbb{P}_{r}^{2}$, having $k$ nodes as its only singularities, that is, $V_{i r r}\left(d ; d_{1}, \ldots, d_{r} ; k\right) \neq \emptyset$.

We prove this in two steps: first, we shall prove the existence of a nonsingular curve in such linear systems on $\mathbb{P}_{r}^{2}$ by means of some modification of the Hirschowitz-criterion (1.2.2), afterwards, we obtain the required nodal curves by a suitable deformation of the union of the previous curve with generic straight lines. 
Corollary 3.1.7. If $r \geq 10$ and positive integers $d ; d_{1}, \ldots, d_{r}$ satisfy

$$
d \geq \sqrt{2} \sqrt{\sum_{i=1}^{r} d_{i}\left(d_{i}+1\right)},
$$

then, for any non-negative integer

$$
k \leq \frac{(d-1)(d-2)}{2}-\sum_{i=1}^{r} d_{i}\left(d_{i}+1\right)
$$

$V_{i r r}\left(d ; d_{1}, \ldots, d_{r} ; k\right) \neq \emptyset$.

This easily follows from Theorem 5 , because $d^{\prime}:=\sqrt{2} \sqrt{\sum_{i=1}^{r} d_{i}\left(d_{i}+1\right)}$ satisfies

$$
\frac{d^{2}+6 d^{\prime}-1}{4}-\frac{d^{\prime}}{2}>\sum_{i=1}^{r} \frac{d_{i}\left(d_{i}+1\right)}{2} .
$$

3.2. Existence of nodal curves on $\mathbb{P}_{r}^{2}, r \leq 9$. As mentioned before, we can restrict to minimal tuples $\left(d ; d_{1}, \ldots, d_{r}\right)$ and construct only rational curves. In the case $r \leq 8$ the statement is, probably, known. Nevertheless, here we provide both, the proof for $r \leq 8$ and $r=9$.

3.2.1. Assume that $r=1$. Clearly, Bézout's theorem implies $d_{1} \leq d-1$ for all curves $C \in V_{i r r}\left(d ; d_{1} ; k\right)$, which are not the strict transform of a line in $\mathbb{P}^{2}$ through $p_{1}$. We take the union of $d_{1}$ distinct straight lines in $\mathbb{P}^{2}$ through $p_{1}$ and $d-d_{1}$ more generic straight lines in $\mathbb{P}^{2}$, lift this curve to $\mathbb{P}_{1}^{2}$ and get a reduced curve in the linear system $\left|d E_{0}-d_{1} E_{1}\right|$ with

$$
\frac{\left(d-d_{1}\right)\left(d-d_{1}-1\right)}{2}+d_{1}\left(d-d_{1}\right)=\frac{(d-1)(d-2)}{2}-\frac{d_{1}\left(d_{1}-1\right)}{2}+d-1
$$

nodes. After smoothing $d-1$ intersection points, we obtain the desired irreducible rational curve.

3.2.2. Assume that $r=2$. Again, by Bézout's theorem, $d_{1}+d_{2} \leq d$ with the exception of the strict transform of the line through $p_{1}$ and $p_{2}$. We consider in $\mathbb{P}^{2}$ the union of $d_{1}$ distinct straight lines through $p_{1}, d_{2}$ distinct straight lines through $p_{2}$ and $d-d_{1}-d_{2}$ additional generic straight lines. The strict transform in $\mathbb{P}_{2}^{2}$ of this curve is a reduced curve in the linear system $\left|d E_{0}-d_{1} E_{1}-d_{2} E_{2}\right|$ with

$$
\begin{aligned}
d_{1} d_{2}+\left(d_{1}+d_{2}\right) & \left(d-d_{1}-d_{2}\right)+\frac{\left(d-d_{1}-d_{2}\right)\left(d-d_{1}-d_{2}-1\right)}{2} \\
= & \frac{(d-1)(d-2)}{2}-\frac{d_{1}\left(d_{1}-1\right)}{2}-\frac{d_{2}\left(d_{2}-1\right)}{2}+d-1
\end{aligned}
$$

nodes. Again one smooths $d-1$ nodes to obtain an irreducible rational curve.

3.2.3. Assume that $r=3$. Due to the minimality condition (3.1.2), we can proceed as follows. In the plane we choose the union of $d_{i}$ distinct straight lines through $p_{i}$, $i=1,2,3$, and $d-d_{1}-d_{2}-d_{3}$ generic straight lines. After smoothing $d-1$ nodes of its strict transform in $\mathbb{P}_{3}^{2}$, as above, we end up with the desired rational nodal curve. 
3.2.4. Assume that $r=4$. By induction on $d$, we shall show that the minimality condition (3.1.2) is sufficient for the existence of a rational irreducible (nodal) curve in $\left|d E_{0}-\sum_{i=1}^{4} d_{i} E_{i}\right|$. In case $d \leq 4$ the only tuples which are not covered by the (preceding) cases $(r \leq 3)$ are $(d ; 1,1,1,1), d \in\{3,4\}$, and $(4 ; 2,1,1,1)$, hence the statement is trivial. If $d \geq 5$ we have

$$
\max _{\#\{j, \ell, n\}=3}\left(\left(d_{j}-1\right)+\left(d_{\ell}-1\right)+\left(d_{n}-1\right)\right) \leq d-2 ;
$$

thus, by the induction assumption, there is an irreducible rational (nodal) curve $C$ in the linear system $\left|(d-2) E_{0}-\sum_{i=1}^{4}\left(d_{i}-1\right) E_{i}\right|$. As is well-known (cf. e.g. [Wae]), a generic (smooth rational) curve $C^{\prime}$ in the one-dimensional base-pointfree linear system $\left|2 E_{0}-\sum_{i=1}^{4} E_{i}\right|$ intersects $C$ transversally. Finally, smoothing one intersection point in the union of $C$ and $C^{\prime}$ completes the induction step.

3.2.5. Assume that $r=5$. We can proceed as in the case $r=4$ with the only exception that in the induction step $C$ will be an irreducible rational nodal curve in $\left|(d-2) E_{0}-d_{1} E_{1}-\sum_{i=2}^{5}\left(d_{i}-1\right) E_{i}\right|$, and $C^{\prime}$ has to be chosen as a generic smooth curve in $\left|2 E_{0}-\sum_{i=2}^{5} E_{i}\right|$. Thereby, obviously, we have to treat the case $\left(d ; d_{1}, \ldots, d_{5}\right)=$ $(d ; d-2,1,1,1,1)$ separately, because there is no irreducible curve in the linear system $\left|(d-2) E_{0}-(d-2) E_{1}\right|$. In this case, we have to choose $C$ as the union of $d-2$ generic lines through $p_{1}$, and to smooth $d-2$ intersection points of $C$ and $C^{\prime}$.

3.2.6. Assume that $r=6$. Again, we construct inductively irreducible rational (nodal) curves only supposing that (3.1.2) holds. First, we consider separately the case $d_{1}=\ldots=d_{6}=1$, where in the induction step a generic line has to be added (and one intersection point smoothed). Then, supposing $d_{1} \geq 2$, in case $d \leq 4$ the only (additionally) possible tuple is $(4 ; 2,1,1,1,1,1)$, where the statement is trivial. For the induction step, we know that

$$
\max \left\{\left(d_{1}-2\right)+\left(d_{2}-1\right)+\left(d_{3}-1\right),\left(d_{2}-1\right)+\left(d_{3}-1\right)+\left(d_{4}-1\right)\right\} \leq d-3 .
$$

Thereby, we can construct the desired curve by smoothing one intersection point in the union of an irreducible nodal rational curve

$$
C \in\left|(d-3) E_{0}-\left(d_{1}-2\right) E_{1}-\sum_{i=2}^{6}\left(d_{i}-1\right) E_{i}\right|
$$

and a generic curve in the one-dimensional (base-point-free) linear system

$$
\left|3 E_{0}-2 E_{1}-E_{2}-E_{3}-E_{4}-E_{5}-E_{6}\right| \text {. }
$$

3.2.7. Assume that $r=7$. Changing $\left(d ; d_{1}, \ldots, d_{r}\right)$ to $\left(d-3 ; d_{1}-1, \ldots, d_{7}-1\right)$ leaves the minimality condition intact. Hence, in the induction step $(d \geq 5)$, we consider the family $\mathcal{F}$ of curves $C \cup C^{\prime}$, where $C$ is a generic rational nodal curve in

$$
\left|(d-3) E_{0}-\sum_{i=1}^{7}\left(d_{i}-1\right) E_{i}\right|
$$

and $C^{\prime}$ is a generic rational nodal curve in $\left|3 E_{0}-E_{1}-\ldots-E_{7}\right|$. First, we consider the only case where $C$ cannot be supposed to be irreducible, namely $\left(d ; d_{1}, \ldots, d_{7}\right)=$ $(d ; d-2,1,1,1,1,1,1)$. In this situation, we proceed as in case $r=5$, take $C$ as the union of $d-3$ generic lines through $p_{1}$ and smooth $d-3$ intersection points of $C$ 
and $C^{\prime}$. Now, we assume $C$ to be irreducible. If $d=5$, then $C$ is a generic curve in one of the following base-point-free linear systems:

$$
\left|2 E_{0}\right|, \quad\left|2 E_{0}-E_{1}\right|, \quad\left|2 E_{0}-E_{1}-E_{2}\right|,
$$

whence a generic member of $\mathcal{F}$ turns out to be a nodal curve. It remains to prove that for $d \geq 6$ a generic member $\tilde{C}$ of the family $\mathcal{F}$ is a nodal curve. Indeed, for the canonical divisor $K_{\mathbb{P}_{7}^{2}}$ we have $\left(K_{\mathbb{P}_{7}^{2}} \cdot C\right)<0$ and $\left(K_{\mathbb{P}_{7}^{2}} \cdot C^{\prime}\right)<0$, hence by ([Nob], Theorem 3.10)

$$
\begin{aligned}
\operatorname{dim} \mathcal{F} & =\left(\frac{(d-3) d}{2}-\frac{(d-4)(d-5)}{2}-\sum_{i=1}^{7}\left(d_{i}-1\right)\right)+\left(\frac{3 \cdot 6}{2}-1-7\right)+1 \\
& =\left(\frac{d(d+3)}{2}-\sum_{i=1}^{7} \frac{d_{i}\left(d_{i}+1\right)}{2}\right)-\left(\frac{(d-1)(d-2)}{2}-\sum_{i=1}^{7} \frac{d_{i}\left(d_{i}-1\right)}{2}\right) \\
& =\operatorname{dim}|\tilde{C}|-\Delta
\end{aligned}
$$

where $\Delta$ denotes the "virtual" number of nodes of $\tilde{C}$. On the other hand, the family of rational curves in $\left|3 E_{0}-E_{1}-\ldots-E_{7}\right|$ has no base point. Hence, the only possibility for a non-nodal singularity of $\tilde{C}$ is a tangency point of $C, C^{\prime}$ with smooth branches. But $\left(K_{\mathbb{P}_{7}^{2}} \cdot C^{\prime}\right)=-2<-1$ and applying ([Nob], Theorem 3.12), in this case we would have had

$$
\operatorname{dim} \mathcal{F}<\operatorname{dim}|\tilde{C}|-\Delta
$$

Finally, smoothing one node in $\tilde{C}$ we get the desired curve.

3.2.8. Assume that $r=8$. Once again, the case $d \leq 4$ is trivial and we suppose $d \geq 5$. In the induction step, we proceed as in the case $r=7$ : We define $\mathcal{F}$ to be the family of curves $C \cup C^{\prime}$, where $C$ is a generic rational nodal curve in

$$
\left|(d-3) E_{0}-\sum_{i=1}^{8}\left(d_{i}-1\right) E_{i}\right|
$$

and $C^{\prime}$ is a generic rational nodal curve in $\left|3 E_{0}-E_{1}-\ldots-E_{8}\right|$. In the case $\left(d ; d_{1}, \ldots, d_{8}\right)=(d ; d-2,1,1,1,1,1,1,1)$, we can repeat the above construction completely. Hence, by the induction assumption, we can assume $C$ to be irreducible. If $d=5$, as in the case $r=7$, a generic member $\tilde{C}$ of $\mathcal{F}$ is a nodal curve. If $d=6$, then $C, C^{\prime}$ can be chosen as the strict transforms of two distinct (irreducible) plane rational cubics through 8 generic points, hence $C \cup C^{\prime}$ is nodal. Let $d \geq 7$. Since the minimality condition implies

$$
\left(K_{\mathbb{P}_{8}^{2}} \cdot C\right)=-3(d-3)+\sum_{i=1}^{8}\left(d_{i}-1\right) \leq-3 d+1+\left[\frac{8}{3} d\right]<-1,
$$

by ([Nob], Theorem 3.10), for dimension reasons the family of rational nodal curves $C \in\left|(d-3) E_{0}-\sum_{i=1}^{8}\left(d_{i}-1\right) E_{i}\right|$ cannot have base points. Hereby, again, the only possibility for a non-nodal singularity of $\tilde{C}$ is a tangency point with smooth branches. Counting dimensions as above, we see that such a singularity may not occur, and we complete the construction as before. 
3.2.9. Assume that $r=9$. By condition (3.1.3) we have only to consider curves of degree $d \geq 4$. We split the induction step into three parts depending on the shape of $d$ :

If $d=3 m+2, m \geq 1$, then the minimality condition (3.1.2) implies

$$
\sum_{i=1}^{9} d_{i} \leq 3 d-3 \quad \text { and } \quad\left(d_{1}>d_{3} \quad \text { or } \quad d_{1}+d_{2}+d_{3} \leq d-1\right) .
$$

In each case, changing $\left(d ; d_{1}, \ldots, d_{9}\right)$ to $\left(d-1 ; d_{1}-1, d_{2}, \ldots, d_{9}\right)$ preserves both (3.1.3) and the minimality condition. Hence, we can assume the existence of an irreducible rational nodal curve $C$ in the linear system

$$
\left|(d-1) E_{0}-\left(d_{1}-1\right) E_{1}-\sum_{i=2}^{9} d_{i} E_{i}\right| .
$$

Adding a generic line through $p_{1}$ and smoothing one intersection point, we obtain the desired curve.

If $d=3 m+1, m \geq 1$, the only case where we have to use another construction is

$$
\left(d ; d_{1}, d_{2}, \ldots, d_{9}\right)=(3 m+1 ; m+1, m, \ldots, m) .
$$

We shall apply the following lemma, which will be proven at the end of this section:

Lemma 3.2.10. Let $L_{i j}$ be the (unique) line in the linear system $\left|E_{0}-E_{i}-E_{j}\right|$, $1 \leq i<j \leq 9$. For any $m \geq 1$ there exists an irreducible rational nodal curve

$$
F_{m} \in\left|3 m E_{0}-m E_{1}-\ldots-m E_{8}-(m-1) E_{9}\right|
$$

which meets every line $L_{i j}, 1 \leq i<j \leq 9$, and the exceptional divisors $E_{1}, \ldots, E_{9}$, transversally and only at non-singular points.

Taking the curve $F_{m}$ from this lemma and applying the base change (3.1.1) in $\operatorname{Pic}\left(\mathbb{P}_{9}^{2}\right)$ with $(j, m, n)=(1,2,9)$, one easily sees that $F_{m}$ belongs to the linear system

$$
\left|(3 m+1) E_{0}^{\prime}-(m+1) E_{1}^{\prime}-(m+1) E_{2}^{\prime}-m E_{3}-\ldots-m E_{8}-m E_{9}^{\prime}\right|,
$$

and is transversal to $E_{2}^{\prime}=L_{19}$. Hence, we get the desired irreducible rational nodal curve in

$$
\left|(3 m+1) E_{0}^{\prime}-(m+1) E_{1}^{\prime}-m E_{2}^{\prime}-m E_{3}-\ldots-m E_{8}-m E_{9}^{\prime}\right|,
$$

by smoothing one intersection point of the nodal curve $F_{m} \cup E_{2}^{\prime}$.

If $d=3 m$, then we have to consider three possibilities

- $d_{1}+d_{2}+d_{3} \leq d-1$,

- $d_{1}>d_{3}$ and $d_{1}+\ldots+d_{9} \leq 3 d-3$,

- $\left(3 m ; m, m, m, d_{4}, \ldots, d_{9}\right)$, where $m \geq d_{4} \geq \ldots \geq d_{9}, d_{9} \leq m-1$.

Clearly, to get the latter curves, it is enough to prove Lemma 3.2.10. In the first two cases we can proceed as in the situation $d=3 m+2$.

Proof of Lemma 3.2.10. We divide our reasoning into several steps.

Step 1. First, we shall show the following. The set of 9 -tuples $\left(p_{1}, \ldots, p_{9}\right) \in\left(\mathbb{P}^{2}\right)^{9}$ such that on the surface $\mathbb{P}^{2}\left(p_{1}, \ldots, p_{9}\right)$ (which is the plane blown up at $p_{1}, \ldots, p_{9}$ )

$$
\left|3 m E_{0}-(m+1) E_{1}-m E_{2}-\ldots-m E_{8}-(m-1) E_{9}\right|=\emptyset
$$


is Zariski-open in $\left(\mathbb{P}^{2}\right)^{9}$, and there is a quasiprojective hypersurface $S \subset\left(\mathbb{P}^{2}\right)^{9}$ such that the linear system (3.2.11) is non-empty and contains an irreducible curve for any $\left(p_{1}, \ldots, p_{9}\right) \in S$. Indeed, assume that $\left(p_{1}, \ldots, p_{9}\right) \in\left(\mathbb{P}^{2}\right)^{9}$ are independent generic points. Applying successively the base change (3.1.1) with

$$
(j, \ell, n)=(1,2,3),(4,5,6),(7,8,9),
$$

respectively, we transform the system (3.2.11) into the system

$$
\left|3(m-1) E_{0}^{\prime}-m E_{1}^{\prime}-(m-1) E_{2}^{\prime}-\ldots-(m-1) E_{8}^{\prime}-(m-2) E_{9}^{\prime}\right| .
$$

After $m-1$ such steps we end up with a system of type

$$
\left|3 E_{0}^{\prime}-2 E_{1}^{\prime}-E_{2}^{\prime}-\ldots-E_{8}^{\prime}\right| \text {. }
$$

In the latter representation, the non-emptiness of the linear system means that the blown-up points $p_{2}^{\prime}, \ldots, p_{8}^{\prime}$ are distinct points on a plane cubic $C_{3}$ with a singularity at $p_{1}^{\prime}$. This, evidently, imposes one non-trivial condition on the points $p_{1}^{\prime}, \ldots, p_{9}^{\prime}$ in contrast to the generality of the initial points $p_{1}, \ldots, p_{9}$. On the other hand, choosing an irreducible cubic $C_{3}$ with a singularity at a point $p_{1}^{\prime}$, arbitrary distinct points $p_{2}^{\prime}, \ldots, p_{8}^{\prime} \in C_{3}$, and $p_{9}^{\prime} \notin C_{3}$, and applying the inverse process of base changes, under the condition that $p_{1}, \ldots, p_{9}$ belong to some hypersurface in $\left(\mathbb{P}^{2}\right)^{9}$, one obtains an irreducible curve in the system (3.2.11), which is unique by Bézout's theorem.

Step 2. In the previous notation, let us specialize the points $p_{1}^{\prime}, p_{4}^{\prime}, p_{9}^{\prime}$ on a line $L^{\prime} \subset \mathbb{P}^{2}$. Applying the base change (3.2.12) in the inverse order to $\mathbb{P}^{2}\left(p_{1}^{\prime}, \ldots, p_{9}^{\prime}\right)$ and blowing down the new exceptional curves, we obtain an irreducible plane sextic curve with a triple point $p_{1}^{\prime \prime}$, double points $p_{2}^{\prime \prime}, \ldots, p_{8}^{\prime \prime}$ and a non-singular point $p_{9}^{\prime \prime}$. Since the operation applied is a composition of three Cremona transformations of $\mathbb{P}^{2}$ with the fundamental points $\left(p_{7}^{\prime}, p_{8}^{\prime}, p_{9}^{\prime}\right),\left(p_{4}^{\prime}, p_{5}^{\prime}, p_{6}^{\prime}\right),\left(p_{1}^{\prime}, p_{2}^{\prime}, p_{3}^{\prime}\right)$, respectively, the points $p_{1}^{\prime \prime}, p_{4}^{\prime \prime}, p_{9}^{\prime \prime}$ lie on a straight line $L^{\prime \prime} \subset \mathbb{P}^{2}$, which corresponds to the strict transform of $L^{\prime}$. We continue in such a manner until we get an irreducible plane curve $C_{3 m}(p)$ of degree $3 m$ with an $(m+1)$-fold point $p_{1}, m$-fold points $p_{2}, \ldots, p_{8}$ and an $(m-1)$-fold point $p_{9}$, such that $p_{1}, p_{4}, p_{9}$ lie on a straight line $L$. By Bézout's theorem, $L$ meets $C_{3 m}(\underline{p})$ transversally at $p_{1}, p_{4}, p_{9}$. Now, fixing $p_{i}^{\prime}, i \neq 4$, we vary the point $p_{4}^{\prime}$ along $C_{3}$. The above construction will give us a one-parametric family of sets $\left\{p_{1}, \ldots, p_{9}\right\}$, thereby a one-parametric (continuous) family of curves $C_{3 m}\left(p_{1}, \ldots, p_{9}\right)$. Generically, the line $L$ will split into three lines $\left(p_{1} p_{4}\right),\left(p_{4} p_{9}\right),\left(p_{1} p_{9}\right)$, which intersect $C_{3 m}(p)$ transversally.

Varying the numbering of $p_{2}^{\prime}, \ldots, p_{8}^{\prime}$, respectively repeating the previous reasoning with the initial specialization of the points $p_{2}^{\prime}, p_{5}^{\prime}, p_{8}^{\prime}$ on a straight line, we obtain, finally, that for a generic element $p=\left(p_{1}, \ldots, p_{9}\right) \in S$, the strict transform of $C_{3 m}(\underline{p})$ intersects each $L_{i j}, 1 \leq i<j \leq 9$, transversally. Since the base change simply interchanges lines $L_{i j}$ with exceptional divisors $E_{s}$, we can claim that the intersection with each exceptional divisor $E_{1}, \ldots, E_{9}$ is transversal as well.

Step 3. The previous statement means, in particular, that for a generic $\underline{p} \in S$ the curve $C_{3 m}(\underline{p}) \subset \mathbb{P}^{2}$ has nine ordinary multiple points and meets every line $\left(p_{i} p_{j}\right)$ transversally. Let us denote by $\mathcal{G}$ the germ at $C_{3 m}(\underline{p})$ of the family of plane rational curves of degree $3 \mathrm{~m}$, having ordinary singular points in a neighbourhood of $p_{2}, \ldots, p_{9}$ with the same multiplicities $m, \ldots, m, m-1$, respectively, and having a point of multiplicity at least $m$ in a neighbourhood $U$ of $p_{1}$. Clearly, $\mathcal{G}$ is the intersection of a germ $\Sigma_{1}$ of the equisingular stratum in $\left|\mathcal{O}_{\mathbb{P}^{2}}(3 m)\right|$, corresponding 
to the ordinary singular points $p_{2}, \ldots, p_{9}$, and a germ $\Sigma_{2}$ of the following family of curves of degree $3 \mathrm{~m}$. A curve in $\Sigma_{2}$ has a point of multiplicity at least $m$ in a neighbourhood $U$ of $p_{1}$ and the sum of $\sigma$-invariants in $U$ is equal to $\frac{1}{2}(m+1) m$. It is not difficult to see that $\Sigma_{2}$ is the union of $m+1$ smooth germs, such that their intersection $\Sigma_{3}$ consists of curves having an ordinary $(m+1)$-fold point in $U$, and a curve in $\Sigma_{2} \backslash \Sigma_{3}$ has in $U$ an ordinary $m$-fold point and $m$ nodes (geometrically, such a deformation looks as if one of the local branches of $C_{3 m}(p)$ at $p_{1}$ moves away from the multiple point). The classical smoothness criteria say that $\Sigma_{1}$ is smooth, and

$$
\operatorname{codim}_{\left|\mathcal{O}_{\mathbb{P} 2}(3 m)\right|} \Sigma_{1} \leq 7\left(\frac{(m+1) m}{2}-2\right)+\left(\frac{m(m-1)}{2}-2\right)=4 m^{2}+3 m-16 .
$$

Since, evidently,

$$
\operatorname{codim}_{\left|\mathcal{O}_{\mathbb{P}^{2}}(3 m)\right|} \Sigma_{2} \leq \frac{(m+1) m}{2}-2+m=\frac{m^{2}}{2}+\frac{3 m}{2}-2,
$$

we obtain

$$
\operatorname{codim}_{\left|\mathcal{O}_{\mathbb{P}^{2}}(3 m)\right|} \mathcal{G} \leq \frac{9 m^{2}}{2}+\frac{9 m}{2}-18 .
$$

On the other hand, we have

$$
\operatorname{dim}\left(\Sigma_{1} \cap \Sigma_{3}\right)=\operatorname{dim} S=17<18 \leq \operatorname{dim}\left(\Sigma_{1} \cap \Sigma_{2}\right) .
$$

That means, there exists a rational plane curve of degree $3 m$ with 8 ordinary $m$-fold points, one ordinary $(m-1)$-fold point and, additionally, $m$ nodes. Moreover, this curve intersects transversally with a straight line through any two of the 9 multiple points. Blowing up these 9 points, we get the desired curve $F_{m} \subset \mathbb{P}_{9}^{2}$.

3.3. Plane curves with generic multiple points. It will be convenient for us to deal here with plane curves having ordinary multiple points instead of nonsingular curves on the blown-up plane. For abuse of language, we shall use the notation: given an ordered set $\underline{p}=\left\{p_{1}, \ldots, p_{r}\right\}$ of distinct points in $\mathbb{P}^{2}$ and an integral vector $\underline{d}=\left(d_{1}, \ldots, d_{r}\right)$, by $S_{d}(\underline{p}, \underline{d})$ we shall denote the set of reduced irreducible curves of degree $d$ which have ordinary singular points at $p_{1}, \ldots, p_{r}$ of multiplicities $d_{1}, \ldots, d_{r}$, respectively, as their only singularities.

Lemma 3.3.1. Let $p_{1}, \ldots, p_{r}, r \geq 1$, be distinct generic points in $\mathbb{P}^{2}$. Then, for any positive integers $d ; d_{1}, \ldots, d_{r}$ satisfying

$$
\frac{d^{2}+6 d-1}{4}-\left[\frac{d}{2}\right]>\sum_{i=1}^{r} \frac{d_{i}\left(d_{i}+1\right)}{2},
$$

there exists a curve $F_{d} \in S_{d}(\underline{p}, \underline{d})$.

Proof. Following [Hir], we shall prove a more general statement. Let $I$ be a subset in $\{1, \ldots, r\}$, let the points $p_{i}, i \in I$, lie on a straight line $G$, and let the points $p_{i}$, $i \notin I$, be in general position outside $G$. If condition (3.3.2) and

$$
\sum_{i \in I} d_{i} \leq d
$$

hold, then there exists a curve $F_{d} \in S_{d}(\underline{p}, \underline{d})$, which is transversal to the line $G$. 
We shall use induction on $d$. If $d=2$ then (3.3.2) reads $2 \geq \sum_{i=1}^{r} d_{i}\left(d_{i}+1\right) / 2$, so the only possibilities are $\left(r=1, d_{1}=1\right)$ or $\left(r=2, d_{1}=d_{2}=1\right)$, when the required curves do exist. Assume $d \geq 3$.

Step 1: Assume that $I=\{1, \ldots, r\}, \sum_{i=1}^{r} d_{i} \leq d$.

If $r=1$ then, by (3.3.2), $d_{1}<d$, hence one obtains the equation of the desired curve in the form

$$
F(x, y):=\sum_{d_{1} \leq i+j \leq d} A_{i j} x^{i} y^{j},
$$

with $p_{1}=(0,0)$ and generic coefficients $A_{i j}$. If $r>1$ then one can obtain the desired curve as a generic member of the linear family of all curves with equations

$$
\lambda^{\prime} C^{\prime} \prod_{i=1}^{r} \prod_{j=1}^{d_{i}} L_{i j}^{\prime}+\lambda^{\prime \prime} C^{\prime \prime} \prod_{i=1}^{r} \prod_{j=1}^{d_{i}} L_{i j}^{\prime \prime}, \quad\left(\lambda^{\prime}: \lambda^{\prime \prime}\right) \in \mathbb{P}^{1},
$$

where for any $1 \leq i \leq r$, we take distinct generic straight lines $L_{i j}^{\prime}, L_{i j}^{\prime \prime}$ through $p_{i}$, $1 \leq j \leq d_{i}$, and $C^{\prime}, C^{\prime \prime}$ are distinct generic curves of degree $d-\sum_{i=1}^{r} d_{i}$.

Step 2: Assume that $\sum_{i=1}^{r} d_{i}>d$ and $\frac{d+2}{2} \leq \sum_{i \in I} d_{i} \leq d$.

Put $\underline{\tilde{d}}:=\left(\tilde{d}_{1}, \ldots, \tilde{d}_{r}\right)$, where $\tilde{d}_{i}=d_{i}-1, i \in I$, and $\tilde{d}_{i}=d_{i}, i \notin I$. Then

$$
\begin{aligned}
\sum_{i=1}^{r} \frac{\tilde{d}_{i}\left(\tilde{d}_{i}+1\right)}{2} & =\sum_{i=1}^{r} \frac{d_{i}\left(d_{i}+1\right)}{2}-\sum_{i \in I} d_{i} \stackrel{(3.3 .2)}{<} \frac{d^{2}+6 d-1}{4}-\left[\frac{d}{2}\right]-\frac{d+2}{2} \\
& \leq \frac{(d-1)^{2}+6(d-1)-1}{4}-\left[\frac{d-1}{2}\right]
\end{aligned}
$$

hence, by the induction assumption, there exists a curve $F_{d-1} \in S_{d-1}(\underline{p}, \underline{\tilde{d}})$, transversal to the line $G$. Put $q:=d-\sum_{i \in I} d_{i}$ and fix $q+1$ distinct generic points $z_{1}, \ldots, z_{q+1}$ on $G$ outside $F_{d-1}$. Since

$$
\sum_{i \in I} d_{i}+q+1=d+1
$$

we have

$$
\begin{aligned}
\sum_{i=1}^{r} \frac{d_{i}\left(d_{i}+1\right)}{2}+q+1 & <\frac{d^{2}+6 d-1}{4}-\left[\frac{d}{2}\right]+\left(d+1-\sum_{i \in I} d_{i}\right) \\
& \leq \frac{d^{2}+6 d-1}{4}-\left[\frac{d}{2}\right]+\frac{d}{2} \leq\left[\frac{(d+3)^{2}}{4}\right]
\end{aligned}
$$

and, according to the Hirschowitz-Criterion (1.2.2),

$$
h^{1}\left(\mathbb{P}^{2}, \mathcal{J}(d)\right)=0
$$

where $\mathcal{J} \subset \mathcal{O}_{\mathbb{P}^{2}}$ is the ideal sheaf defined by

$$
\mathcal{J}_{p_{i}}=\left(\mathfrak{m}_{p_{i}}\right)^{d_{i}}, \quad i=1, \ldots, r, \quad \mathcal{J}_{z_{j}}=\mathfrak{m}_{z_{j}}, \quad j=1, \ldots, q+1 .
$$

That means that

$$
\begin{aligned}
h^{0}\left(\mathbb{P}^{2}, \mathcal{J}(d)\right) & =h^{0}\left(\mathbb{P}^{2}, \mathcal{O}_{\mathbb{P}^{2}}(d)\right)-\sum_{i=1}^{r} \operatorname{dim} \mathcal{O}_{\mathbb{P}^{2}, p_{i}} /\left(\mathfrak{m}_{p_{i}}\right)^{d_{i}}-\sum_{j=1}^{q+1} \operatorname{dim} \mathcal{O}_{\mathbb{P}^{2}, z_{j}} / \mathfrak{m}_{z_{j}} \\
& =\frac{(d+1)(d+2)}{2}-\sum_{i=1}^{r} \frac{d_{i}\left(d_{i}+1\right)}{2}-q-1
\end{aligned}
$$


or, in other words, the

$$
m:=\sum_{i=1}^{r} \frac{d_{i}\left(d_{i}+1\right)}{2}+q+1
$$

linear conditions on curves of degree $d$ imposed by the multiple points $p_{1}, \ldots, p_{r}$, $z_{1}, \ldots, z_{q+1}$ are independent. We write these conditions as linear equations $\Lambda_{j}(F)=$ $0,1 \leq j \leq m$, in the coefficients of a curve $F$ of degree $d$, such that $\Lambda_{m}(F)=0$ expresses the passage of $F$ through $z_{q+1}$. Due to the above independence, we find a curve $F \in H^{0}\left(\mathcal{O}_{\mathbb{P}^{2}}(d)\right)$ satisfying

$$
\Lambda_{1}(F)=\ldots=\Lambda_{m-1}(F)=0, \quad \Lambda_{m}(F)=1 .
$$

Let us consider the linear family $\lambda F_{d-1} G+\mu F,(\lambda: \mu) \in \mathbb{P}^{1}$. By Bertini's theorem and by the construction of $F_{d-1}$ and $F$, the generic member $F_{\lambda, \mu}$ of this family is irreducible and belongs to $S_{d}(p, \underline{d})$. The only thing we need to show, is the transversality of $F_{\lambda, \mu}$ and $G$. By construction, $F_{\lambda, \mu}$ has multiplicities $d_{i}$ at $p_{i}, i \in I$, respectively, and contains $q=d-\sum_{i \in I} d_{i}$ extra points $z_{1}, \ldots, z_{q}$ on $G$, hence, clearly, $F_{\lambda, \mu}$ and $G$ meet transversally.

Step 3: Assume that $\sum_{i=1}^{r} d_{i}>d, d_{1} \geq \frac{d+2}{2}$ and $\sum_{i \in I} d_{i} \leq \frac{d+1}{2}$.

Define $\underline{\tilde{d}}:=\left(d_{1}-1, d_{2}, \ldots, d_{r}\right)$. As is $(3.3 .3)$, we obtain

$$
\sum_{i=1}^{r} \frac{\tilde{d}_{i}\left(\tilde{d}_{i}+1\right)}{2}<\frac{(d-1)^{2}+6(d-1)-1}{4}-\left[\frac{d-1}{2}\right] .
$$

Hence, there exists a curve $F_{d-1} \in S_{d-1}(\underline{p}, \underline{\tilde{d}})$, transversal to $G$. Note that

$$
\begin{aligned}
\operatorname{dim} S_{d-1}(\underline{p}, \underline{\tilde{d}}) & \geq \frac{(d-1)(d+2)}{2}-\frac{\left(d_{1}-1\right) d_{1}}{2}-\sum_{i=2}^{r} \frac{d_{i}\left(d_{i}+1\right)}{2} \\
& >\frac{(d-1)(d+2)}{2}-\frac{(d-1)^{2}+6(d-1)-1}{4}+\left[\frac{d-1}{2}\right] \\
& =\frac{d^{2}-2 d+2}{4}+\left[\frac{d-1}{2}\right] \geq 2,
\end{aligned}
$$

as $d \geq 3$. Therefore we find a curve $\tilde{F}_{d-1} \in S_{d-1}(\underline{p}, \underline{\tilde{d}})$, linearly independent of $F_{d-1}$. Consider the linear family

$$
\lambda F_{d-1} L+\mu \tilde{F}_{d-1} \tilde{L}, \quad(\lambda: \mu) \in \mathbb{P}^{1},
$$

where $L, \tilde{L}$ are distinct generic straight lines through $p_{1}$. Then, by Bertini's theorem a generic member of this family is irreducible, belongs to $S_{d}(\underline{p}, \underline{d})$ and is transversal to $G$.

Step 4: Assume that $d$ is odd and $\sum_{i \in I} d_{i}=\frac{d+1}{2}=d_{m}:=\max \left\{d_{i} \mid i \notin I\right\}$.

First, we show that $\#(I)>1$. Indeed, otherwise, we would have

$$
0 \stackrel{(3.3 .2)}{<} \frac{d^{2}+6 d-1}{4}-\frac{d-1}{2}-\frac{d+1}{2}\left(\frac{d+1}{2}+1\right)=-\frac{1}{2} .
$$

Choose $j \neq k \in I$. Since $d_{m}+\min \left\{d_{j}, d_{k}\right\} \geq \frac{d+2}{2}$ and $d_{m}+\max \left\{d_{j}, d_{k}\right\} \leq d$, once again as in (3.3.3), we obtain for

$$
\begin{array}{ll}
\underline{\tilde{d}}=\left(\tilde{d}_{1}, \ldots, \tilde{d}_{r}\right), & \tilde{d}_{i}:=d_{i} \text { for } i \notin\{m, j\}, \quad \tilde{d}_{i}:=d_{i}-1 \text { for } i \in\{m, j\}, \\
\underline{\tilde{d}^{\prime}}=\left(\tilde{d}_{1}^{\prime}, \ldots, \tilde{d}_{r}^{\prime}\right), \quad \tilde{d}_{i}^{\prime}:=d_{i} \text { for } i \notin\{m, k\}, \quad \tilde{d}_{i}^{\prime}:=d_{i}-1 \text { for } i \in\{m, k\},
\end{array}
$$


the existence of curves $\tilde{F}_{d-1} \in S_{d-1}(\underline{p}, \underline{\tilde{d}}), \tilde{F}_{d-1}^{\prime} \in S_{d-1}\left(\underline{p}, \underline{\tilde{d}}^{\prime}\right)$, transversal to $G$. Let $\tilde{G}$ be the straight line through $p_{m}, p_{j}$, and $\tilde{G}^{\prime}$ be the straight line through $p_{m}$, $p_{k}$. Consider the linear family

$$
\tilde{\lambda} \tilde{F}_{d-1} \tilde{G}+\tilde{\lambda}^{\prime} \tilde{F}_{d-1}^{\prime} \tilde{G}^{\prime}, \quad\left(\tilde{\lambda}: \tilde{\lambda}^{\prime}\right) \in \mathbb{P}^{1} .
$$

By Bertini's Theorem, a generic member $F$ of this family has only the singular points $p_{1}, \ldots, p_{r}$ of multiplicities $d_{1}, \ldots, d_{r}$, respectively, and is transversal to $G$. Since $\tilde{F}_{d-1} \tilde{G}$ has ordinary singularities at $p_{i}, i \notin\{j, m\}$, and $\tilde{F}_{d-1}^{\prime} \tilde{G}^{\prime}$ has ordinary singularities at $p_{i}, i \notin\{k, m\}, F$ has ordinary singularities at $p_{i}, i \neq m$. At the point $p_{m}$, the curve $\tilde{F}_{d-1} \tilde{G}$ has at most one multiple tangent, which should coincide with $\tilde{G}$, on the other hand $\tilde{F}_{d-1}^{\prime} \tilde{G}^{\prime}$ has at most one multiple tangent, which should coincide with $\tilde{G}^{\prime} \neq \tilde{G}$. Therefore, $F$ has no multiple tangent at $p_{m}$. Finally we have to show that $F$ is irreducible, but this follows immediately from Bertini's theorem: indeed, the only possibility for $F$ to be reducible is $F=F_{d-1} L$, where $F_{d-1}$ is an irreducible curve of degree $d-1$ and $L$ is a straight line, which must vary from $\tilde{G}$ to $\tilde{G}^{\prime}$ as $\left(\tilde{\lambda}: \tilde{\lambda^{\prime}}\right)$ runs through $\mathbb{P}^{1}$, which is impossible, because $F_{d-1}$ must have multiplicity $d_{j}$ at $p_{j}$ and multiplicity $d_{k}$ at $p_{k}$, while $\tilde{F}_{d-1}$ has multiplicity $d_{j}-1$ at $p_{j}$.

Step 5: Assume that $\sum_{i=1}^{r} d_{i}>d, \sum_{i \in I} d_{i} \leq \min \left\{\frac{d+1}{2}, d-d_{m}\right\}$ and $d_{m} \leq \frac{d+1}{2}$. (Again, $d_{m}$ denotes $\max \left\{d_{i} \mid i \notin I\right\}$.) In this case, we specialize the point $p_{m}$ to a generic point in $G \backslash\left\{p_{i} \mid i \in I\right\}$, and we end up with one of the cases 2-5.

Finally, consider for each $r$-tuple $\left(p_{1}, \ldots, p_{r}\right)$ of points in $\mathbb{P}^{2}$ the non-empty linear system of curves with multiplicity at least $d_{i}$ at $p_{i}(1 \leq i \leq r)$. Then in each of the occurring cases the Hirschowitz-Criterion (1.2.2) implies the non-speciality. Hence such linear systems are equidimensional and give an irreducible variety. But the condition for being irreducible with only given non-degenerate multiple points is open (it is described by inequalities); hence the existence of such a curve in a more special situation (as considered above) implies the existence in the original situation.

3.4. Construction of nodal curves. Given $k, d, d^{\prime}, d_{1}, \ldots, d_{r}$ satisfying conditions (3.1.5) and (3.1.6), we shall construct a reduced irreducible plane curve of degree $d$ with ordinary singular points $p_{1}, \ldots, p_{r}$ of multiplicities $d_{1}, \ldots, d_{r}$, respectively, and with $k$ additional nodes as its only singularities.

Let us fix distinct generic points $p_{1}, \ldots, p_{r} \in \mathbb{P}^{2}$, take an irreducible curve $\Phi \in$ $S_{d^{\prime}}(\underline{p}, \underline{d})$, and put

$$
F:=\Phi \prod_{i=1}^{d-d^{\prime}} L_{i}
$$

where $L_{1}, \ldots, L_{d-d^{\prime}}$ are distinct generic straight lines. This curve $F$ has ordinary singular points $p_{1}, \ldots, p_{r}$ of multiplicities $d_{1}, \ldots, d_{r}$, respectively, and $m:=$ $d(d-1) / 2-d^{\prime}\left(d^{\prime}-1\right) / 2$ nodes $z_{1}, \ldots, z_{m}$ as its only singularities. We shall show that it is possible to smooth prescribed nodes keeping the given ordinary singularities and the rest of the nodes, and thus prove Theorem 5 .

As we have seen above, we can deduce the required independence of the deformations of the nodes from

$$
H^{1}\left(F, \mathcal{N}_{F / \mathbb{P}^{2}}^{\prime}\right)=0,
$$


$\mathcal{N}_{F / \mathbb{P}^{2}}^{\prime}:=\operatorname{Ker}\left(\mathcal{N}_{F / \mathbb{P}^{2}} \rightarrow \mathcal{T}_{F}\right)$, where $\mathcal{T}_{F}$ denotes the skyscraper sheaf on $\mathbb{P}^{2}$ concentrated at $p_{1}, \ldots, p_{r}, z_{1}, \ldots, z_{m}$ defined by

$$
\mathcal{T}_{F, p_{i}}:=\mathcal{O}_{\mathbb{P}^{2}, p_{i}} /\left(\mathfrak{m}_{p_{i}}\right)^{d_{i}}, \quad 1 \leq i \leq r, \quad \mathcal{T}_{F, z_{j}}:=\mathcal{O}_{\mathbb{P}^{2}, z_{j}} / \mathfrak{m}_{z_{j}}, \quad 1 \leq j \leq m .
$$

We prove (3.4.1) by induction on $d$. If $d=d^{\prime}$, then the vanishing of $H^{1}\left(F, \mathcal{N}_{F / \mathbb{P}^{2}}^{\prime}\right)$ is provided by the Hirschowitz-Criterion (1.2.2), because (3.1.5) implies

$$
\left[\frac{\left(d^{\prime}+3\right)^{2}}{4}\right]>\sum_{i=1}^{r} \frac{d_{i}\left(d_{i}+1\right)}{2} .
$$

Assume that $d>d^{\prime}$. Then denote

$$
\tilde{F}:=\Phi \prod_{i=1}^{d-d^{\prime}-1} L_{i}
$$

that is, $F=\tilde{F} L_{d-d^{\prime}}$. Let $\tilde{F} \cap L_{d-d^{\prime}}:=\left\{z_{1}, \ldots z_{l}\right\}$, then $\mathcal{T}_{\tilde{F}}$ denotes the restriction of $\mathcal{T}_{F}$ to $\left\{p_{1}, \ldots, p_{r}, z_{l+1}, \ldots, z_{m}\right\}$. Consider the exact sequence

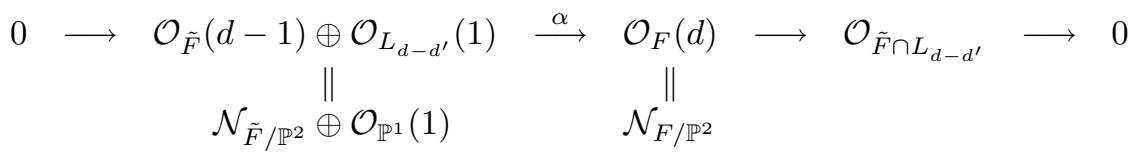

where $\alpha$ is given by $\operatorname{id}_{1} \otimes L_{d-d^{\prime}}+\tilde{F} \otimes \mathrm{id}_{2}$. Clearly, $\alpha$ maps $\mathcal{N}_{\tilde{F} / \mathbb{P}^{2}}^{\prime} \oplus \mathcal{O}_{\mathbb{P}^{1}}(1)$ to $\mathcal{N}_{F / \mathbb{P}^{2}}^{\prime}$. Therefore, (3.4.1) follows from the induction assumption

$$
H^{1}\left(\tilde{F}, \mathcal{N}_{\tilde{F} / \mathbb{P}^{2}}^{\prime}\right)=0,
$$

and we are finished.

\section{REFERENCES}

[ArC] Arbarello, E.; Cornalba, M.: Footnotes to a paper of Beniamino Segre. Math. Ann. 256, 341-362 (1981). MR 83d:14016

[GrK] Greuel, G.-M.; Karras, U.: Families of varieties with prescribed singularities. Comp. Math. 69, 83-110 (1989). MR 90d:32037

[GrL] Greuel, G.-M.; Lossen, C.: Equianalytic and equisingular families of curves on surfaces. Manuscr. Math. 91, 323-342 (1996). CMP 97:3

[GrM] Gradolato, M.A.; Mezzetti, E.: Families of curves with ordinary singular points on regular surfaces. Ann. Mat. Pura et Appl. 150, 281-298 (1988). MR 89g:14015

[Har] Harris, J.: On the Severi problem. Invent. Math. 84, 445-467 (1985) MR 87f:14012

$[\mathrm{HiF}]$ Hirzebruch, F.: Singularities of algebraic surfaces and characteristic numbers. Contemp. Math. 58, 141-155 (1986). MR 87j:14057

[Hir] Hirschowitz, A.: Une conjecture pour la cohomologie des diviseurs sur les surfaces rationelles génériques. J. Reine Angew. Math. 397, 208-213 (1989). MR 90g:14021

[Ivi] Ivinskis, K.: Normale Flächen und die Miyaoka-Kobayashi-Ungleichung, Diplomarbeit, Univ. Bonn (1985).

[Nob] Nobile, A.: Families of curves on surfaces. Math. Zeitschrift 187, 453-470 (1984). MR 85i: 14017

[Ran] Ran, Z.: Families of plane curves and their limits: Enriques' conjecture and beyond. Annals of Math. 130, no.1, 121-157 (1989). MR 90e:14024

[Sev] Severi, F.: Vorlesungen über algebraische Geometrie. Teubner (1921) resp. Johnson (1968).

[Sh1] Shustin, E.: Real plane algebraic curves with prescribed singularities. Topology 32 845-856 (1993). MR 95f:14049

[Sh2] Shustin, E.: Geometry of equisingular families of plane algebraic curves. J. Algebr. Geom. 5, 209-234 (1996). CMP 96:8

[Ta1] Tannenbaum, A.: Families of algebraic curves with nodes. Compositio Math. 41, 107-126 (1980). MR 82b:14017 
[Ta2] Tannenbaum, A.: Families of curves with nodes on K3-surfaces. Math. Ann. 260, 239-253 (1982). MR 84g:14025

[Var] Varchenko, A.N.: Asymptotics of integrals and Hodge structures, (Russian), Modern Problems of Math., Vol. 22, 130-166 (1983); English transl. in Soviet Math. Z. 27 (1984), no. 3. MR 85j:32012

[Wae] van der Waerden, B.L.: Einführung in die algebraische Geometrie, 2nd edition, Springer (1973). MR 49:8984

[Zar] Zariski, O.: Dimension theoretic characterization of maximal irreducible algebraic systems of plane nodal curves of a given order $\mathrm{n}$ and with a given number $d$ of nodes. Amer. J. Math. 104, 209-226 (1982). MR 83m:14044

Universität Kaiserslautern, Fachbereich Mathematik, Erwin-Schrödinger-Strasse, D - 67663 Kaiserslautern, Germany

E-mail address: greuel@mathematik.uni-kl.de

Universität Kaiserslautern, Fachbereich Mathematik, Erwin-Schrödinger-Strasse, D - 67663 Kaiserslautern, German

E-mail address: lossen@mathematik.uni-kl.de

Tel Aviv University, School of Mathematical Sciences, Ramat Aviv, Tel Aviv 69978, ISRAEL

E-mail address: shustin@math.tau.ac.il 